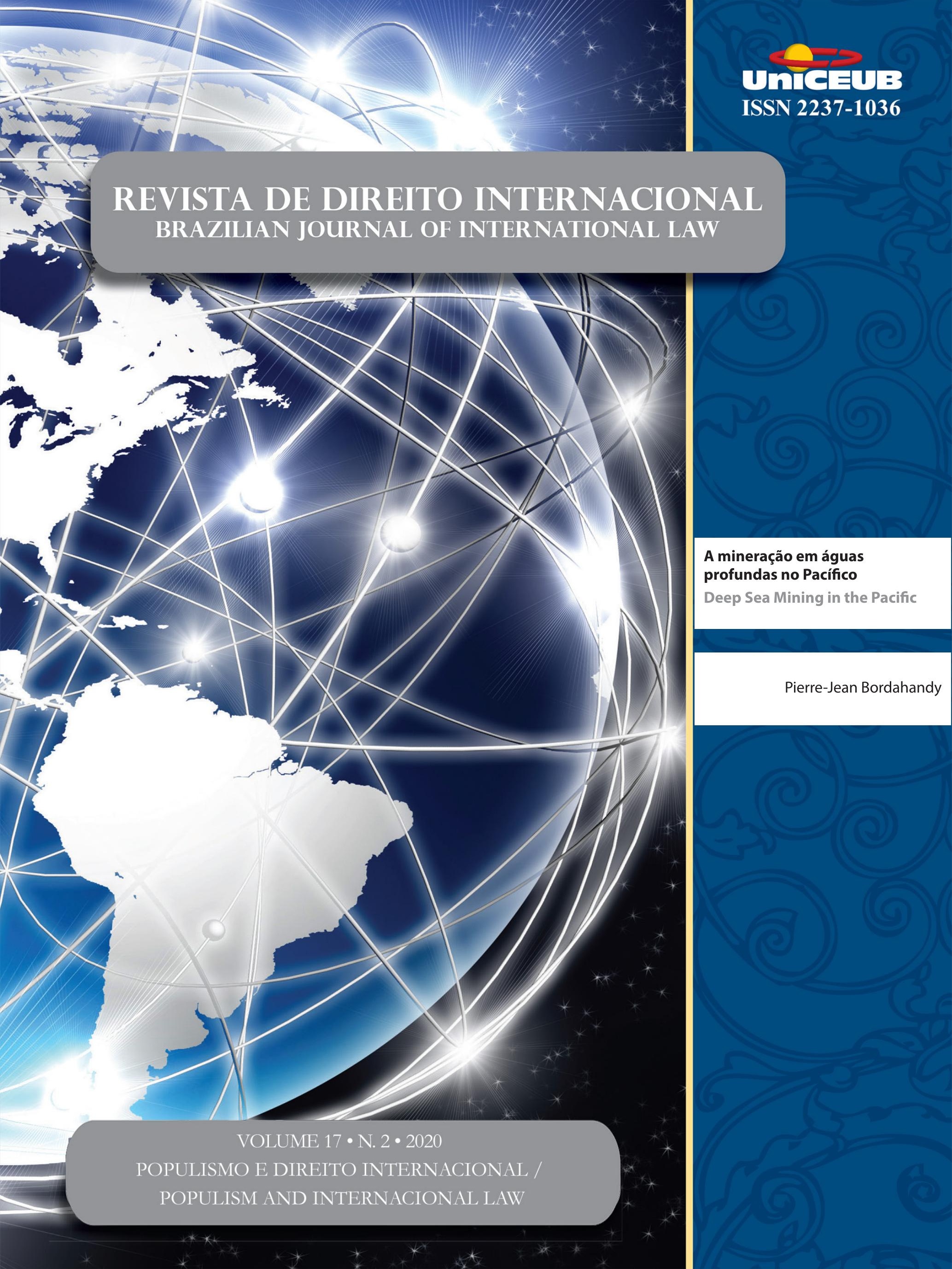




\section{Sumário}

I. CRônicas

A Favor de uma Corte Latino-Americana de Justiça ..................................................... 3 Nitish Monebhurrun

Sobre A SOluÇão JUdicial de CONTROvérsias Na AMÉRICA LATINA 7 Lucas Carlos Lima

A Favor de uma Corte Latino-Americana de Justiça - Uma Reação sobre a Legalidade e A Legitimidade De Seu Desenho Institucional............................................................15

Arthur Roberto Capella Giannattasio

O Estudo do Direito Internacional sob uma Nova Perspectiva: Nossa Experiência na Philip C. Jessup International Moot Court Competition............................................20 Ana Vitória Muniz Bokos, Igor Medeiros Maia, Jefferson Seidy Sonobe Hable, Gabriel de Oliveira Borba, Gilda Nogueira Paes Cambraia e Nayara Lima Rocha Da Cruz

Revisión de laudos de arbitrajes de inversión 2019: I Encuentro Anual (Santiago de

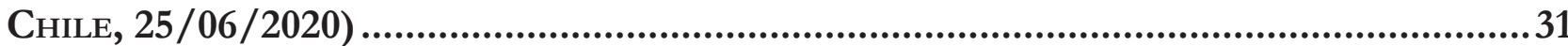
Andrés Delgado Casteleiro e Ivette Esis

II. Dossiê: Populismo e Direito Internacional / Populism and Internatio-

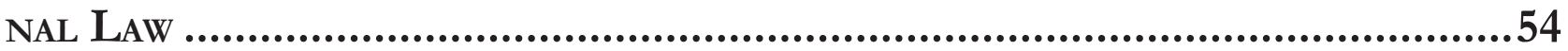

Editorial: Populism and International Law: Global South Perspectives .56 Lucas Lixinski e Fabio Morosini

Editorial: Populismo e Direito Internacional: Perspectivas do Sul Global .61 Lucas Lixinski e Fabio Morosini 
Between Science and populism: the Brazilian Response to COVID-19 from the Perspective of the legal determinants of Global Health

Deisy de Freitas Lima Ventura e Jameson Martins

Populism, ENVIronmental LAW, AND THE POST-PANDEMiC ORDER Alessandra Lehmen

Populism and the Evangelical church in Latin America: how anti-LGBTI forces TRIED TO STOP THE COLOMBIAN PEACE AGREEMENT Julia Assmann de Freitas Macedo e Fabrízio Conte Jacobucci

“Deus EM Davos": o Direito INTERnACIONAL ENTRE REACIONÁRIOS E NEOLIBERAIS NO GOVERNO BOLSONARO 121

Lucas Tasquetto e João Roriz

CHINESE POPULISM IN THE 1920s, EXTRATERRITORIALITY AND INTERNATIONAL LAW 139 Wanshu Cong

CONCEPTUALIZING UNILATERALISM, FRAGMENTATIONISM AND STATISM IN A POPULISM CONTEXT: A RISE OF POPULIST INTERNATIONAL LAW? 162 Wei Shen e Carrie Shu Shang

AUTONOMÍA ADMINISTRATIVA SIN AUTONOMÍA POLÍTICA: LA APLICACIÓN DEL MODELO "UN PAÍS dos SISTEMAs" eN HoNg Kong 186 Juan Enrique Serrano Moreno

III. Artigos sobre outros temas...................................................... 197

Um TWAiler entre nós? As contribuições de Celso Duvivier de Albuquerque Mello PARA O DIREITO INTERNACIONAL (CRÍTICO) NO BRASIL .................................................... 199

Fabio Morosini e Matheus Leichtweis

DEMOCRACIES IN DANGER: ARE JUDICIAL DIALOGUES MEANS TO REFRAIN SETBACKS IN LATIN America?.

Melina Girardi Fachin e Bruna Nowak 


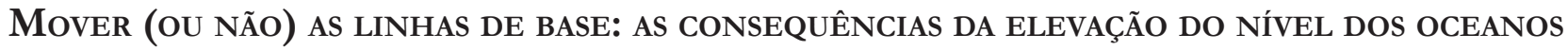
SOBRE AS ZONAS MARÍTIMAS DOS PEQUENOS ESTADOS INSULARES EM DESENVOLVIMENTO E AS ALTERNATIVAS JURÍDICAS PARA REDUZIR SEUS IMPACTOS

Alexandre Pereira da Silva

A mineração em águas profundas no Pacífico 263 Pierre-Jean Bordahandy

Dual Nationality and International Law in times of Globalization. Challenges and Opportunities for Consular Assistance and Diplomatic Protection in RECENT CASES. 288

Walter Arevalo-Ramirez e Robert Joseph Blaise Maclean

Expulsion of aliens: the application of International Law by Chilean Superior Courts 309

Regina Ingrid Díaz Tolosa

O TIPO PENAL BRASILEIRO DE PROMOÇÃo DE MIGRAÇÃO ILEGAL E O PRINCÍPIO DA NÃO CRIMINALIZAÇÃo DA MOBILIDADE HUMANA

Regina Cândido Lima e Silva Santos e Deilton Ribeiro Brasil

INDICADORES TRANSNACIONAIS DE CORRUPÇÃO AMBIENTAL: A OPACIDADE NA TRANSPARÊNCIA INTERNACIONAL

Márcio Ricardo Staffen

SubTRAÇÃo INTERNACIONAL DE CRIANÇAS: ANÁLISE DAS EXCEÇÕES AO RETORNO IMEDIATO DO MENOR À RESIDÊNCIA HABITUAL E CRÍTICA AO ENQUADRAMENTO DA VIOLÊNCIA DOMÉSTICA COMO FLEXIBILIDADE PERMISSIVA

Vivian Daniele Rocha Gabriel

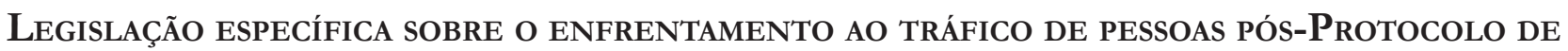
Palermo (2000): análise do Estado de Moçambique 383

Mercia Cardoso de Souza, Guirino Dinis José Nhatave e Francisco Horácio da Silva Frota 
IV. Artigos traduzidos

DIREITO INTERNACIONAL DO RECONHECIMENTO

Emmanuelle Tourme-Jouannet e Tradutor: Ademar Pozzatti Junior

V. RESENHAS

Fabio Costa Morosini, Gabriel Lee Mac Fadden Santos, Valentina Fonseca da Luz e Vinicius Tejadas Maia 


\title{
A mineração em águas profundas no Pacífico*
}

\author{
Deep Sea Mining in the Pacific
}

Pierre-Jean Bordahandy**

\section{Resumo}

De modo geral, este artigo analisa a atividade de mineração em águas profundas no Pacífico sob os vários espectros da economia azul, do desenvolvimento sustentável dos Estados do Pacífico, bem como os ganhos financieros e as perdas ambientais potenciais que podem resultar dessa atividade. Mais especificamente, este artigo se concentra na comparação das diferenças de enquadramento jurídico entre o DSM na área dentro das jurisdições nacionais e em áreas além da jurisdição nacional, com o objetivo de destacar algumas questões legais, estruturais e práticas agudas que devem ser tratadas antes da realização real de qualquer atividade de exploração e pesquisa aplicada ou de exploração. Em última análise, este artigo argumenta por uma consistência estreita entre os dois tipos de regimen jurídicoss (dentro e fora da jurisdição nacional), com uma preferência pelo regime da "área" como modelo para ambos. Implicitamente, este artigo também pondera a relevância de uma extensão da jurisdição do ISA para a coluna de água acima da área.

Palavras chave: Mineração. Águas profundas. Pacífico

\section{Abstract}

Overall, this paper looks at the activity of Deep Sea Mining in the Pacific under the various spectrums of the blue economy, the sustainable development of Pacific States, as well as the gains and potential environmental loss that could flow from this activity. More specifically, this paper focus on comparing the legal framework differences between DSM in area within national jurisdictions and in areas beyond national jurisdiction with the view of highlighting some acute legal, structural and practical issues that should be dealt with before the actual realisation of any DSM exploration and applied research or exploitation projects. Ultimately this paper advocate for a close consistency between the two kinds of legal frameworks (within and beyond national jurisdiction) with a preference for the regime of "the area" to be the template for both. Implicitly, this paper also ponders the relevance of an extension of the ISA jurisdiction to the water column above the area.

Keywords: Mining. Deep Sea Mining. Pacific 


\section{Introdução}

"Para quem somente sabe usar martelo, todo problema é um prego". É em referência a esse provérbio que o pesquisador americano Dennis Meadows, um dos quatro contribuidores do relatório sobre os limites do crescimento, encomendado pelo Clube de Roma ao Massachusetts Institute of Technology (MIT) em 1972, ${ }^{1}$ já denunciava o problema da busca de um crescimento infinito com um conjunto de recursos terrestres esgotáveis. ${ }^{2}$

O fato de buscar novos eldorados potenciais como o mundo marinho, nesse caso a mineração em águas profundas (Deep Sea Mining - DSM - em inglês) ${ }^{3}$, para satisfazer as necessidades intermináveis de crescimento, não altera a análise decorrente do relatório de 1972 supracitado.

Deparamo-nos, assim, com essa noção de limite e de necessidade de gerenciamento dos recursos como fio condutor do último relatório da Comissão Europeia sobre a Economia Azul ${ }^{4}$ que trata incidentalmente do DSM. ${ }^{5}$ A Economia Azul está presente nesse relatório como um conceito, aborda a economia das atividades marítimas e tem por objetivo a exploração "sustentável" do oceano de forma muito ampla. Com esse enfoque, a integralidade da cadeia de extração, de transporte e de tratamento do minério extraído em DSM seria englobada na Economia Azul.

\footnotetext{
MEADOWS, D. H.; MEADOWS, D.; Jørgen, R.; \& BEHRENS III, W. W. The Limits to Growth. The Club of Rome, A Report for the Club of Rome's Project on the Predicament of Mankind. New York: Universe Books, 1972. Reeditado em 26 de Novembro 2017. ISBN 0876631650. Disponível em: < http://www.donellameadows. org/wp-content/userfiles/Limits-to-Growth-digital-scan-version. pdf $>$. Acesso em: 15 out. 2018.

2 MEADOWS, Dennis. A brief and incomplete history of operational gaming in system dynamics. System Dynamics Review, 23 (Issue 2-3), 199-203, Ed. J. D. Sterman, 2007.Disponível em: < https://www.systemdynamics.org/assets/docs/sd_games_history. pdf $>$. Acesso em: 18 out. 2018.

3 O acrônimo inglês DSM (Deep Sea Mining) será utilizado por comodidade neste estudo.

4 COMMISSION EUROPÉENNE. The 2018 Annual Economic Report on Blue Economy. Direction Générale des affaires maritimes et de la pêche. Vol. 01, Catálogo KL-AR-18-001-EN-N, 2018. ISBN 978-92-79-81757-1, ISSN 2599-6584. DOI 10.2771/305342. Disponível em: <https://publications.europa.eu. /en/publicationdetail/-/publication/79299d10-8a35-11e8-ac6a-01aa75ed71a1>. Acesso em: 15 out. 2018.

5 «The 2018 Annual Economic Report on Blue Economy» op. cit. p. 78 .
}

Contudo, é surpreendente encontrar o DSM figurando em um relatório sobre a Economia Azul uma vez que o elo entre essa atividade mineira e o desenvolvimento "durável" pode parecer tênue do ponto de vista das consequências presumíveis, mesmo se não comprovadas, para o meio ambiente, como será mencionado em seguida. No entanto, segundo Gunter Pauli, ${ }^{6}$ um dos principais arquitetos senão o pai desse conceito, a durabilidade faz parte da própria essência da Economia Azul.

Do mesmo modo, possivelmente ainda mais crítico, o relatório conjunto do Banco Internacional para a reconstrução e o desenvolvimento e do Banco Mundial sobre a "Economia Azul" de 2017 enfatiza bem sobre a necessidade do desenvolvimento sustentável nesta área. ${ }^{7}$ Esse relatório lança, sobretudo, um alerta inequívoco em relação às operações de DSM. ${ }^{8}$

Não se trata de uma mensagem isolada, pois o Banco Mundial já havia insistido sobre a necessidade de adotar o Princípio de Precaução na área de DSM ${ }^{9}$, especialmente para os pequenos Estados Insulares em Desenvolvimento (Small Island Developping Countries - SIDS).

Desse modo, os riscos do DSM, com relação ao desenvolvimento durável, não podem ser ignorados, sejam

PAULI Gunter. The Blue Economy: 10 Years, 100 Innovations, 100 Million Jobs. The Club of Rome. Taos, New Mexico USA: Paradigm Publications, 2010. ISBN 978-0-912111-90-2.

THE WORLD BANK (UNITED NATIONS DEPARTMENT OF ECONOMIC AND SOCIAL AFFAIRS). The Potential of the Blue Economy : Increasing Long-term Benefits of the Sustainable Use of Marine Resources for Small Island Developing States and Coastal Least Developed Countries. The World Bank, International Bank for Reconstruction and Development. Washington DC: World Bank, 2017.Disponível em: <https://openknowledge.worldbank.org/bitstream/handle/10986/26843/115545. pdf? sequence $=1$ \&isAllowed $=\mathrm{y}>$. Acesso em: 10 abril 2019 .

8 INTERNATIONAL BANK FOR RECONSTRUCTION AND DEVELOPMENT \& THE WORLD BANK. Ibid: "For these and other reasons, and in the context of an extensive review of the potential for economic development of Pacific Island countries by 2040, a new World Bank report recommends that countries supporting or considering deep-sea mining (DSM) activities proceed with the highest degree of caution to avoid irreversible damage to the ecosystem and that they ensure that appropriate social and environmental safeguards are in place as part of strong governance arrangements for this emerging industry. The study highlights the need to take a precautionary approach, particularly in view of the potential impacts on marine living resources upon which these states depend almost exclusively (o estudo em referência emana do relatório citado abaixo)", 2017.

9 THE WORLD BANK. Pacific Possible. Precautionary Management of Deep Sea Mining Potential in Pacific Island Countries. Washington DC: The World Bank, 2016.Disponível em: <http://pubdocs.worldbank.org/en/125321460949939983/ Pacific-Possible-Deep-Sea-Mining.pdf> . Acesso em: 10 abril 2019. 
eles minimizados, apresentados como reais e complexos a serem identificados ou salientados. Se não é incongruente abordar os riscos antes mesmo de introduzir propriamente dito a atividade de Deep Sea Mining (DSM), convém, a esse ponto, fornecer algumas informações de ordem geral sobre o que o DSM abrange antes de nos aprofundarmos no quadro jurídico no qual se inscreve essa atividade.

As explicações que seguem têm como objetivo demonstrar porque das empresas de DSM perdura apesar dos riscos evocados acima e das dificuldades de extração em águas profundas. A título de breve apresentação, diríamos que o DSM cobre de fato três tipos de depósitos:

1. os depósitos de nódulos polimetálicos localizados entre 4000 e 6500 metros de profundidade.

2. Os depósitos metalíferos de sulfuretos maciços localizados entre 1500 e 5000 metros de profundidade.

3. Os depósitos de crostas metalíferas entre 400 e 4000 metros de profundidade.

O interesse do DSM reside no fato de que as concentrações em minério são nitidamente superiores em depósitos no mar que na terra, consoante Quadro 1. O DSM oferece assim a perspectiva de uma mineração mais rentável, sob-reserva de poder proceder a uma extração de menor custo, o que não é evidente tendo em conta as profundidades evocadas.

Quadro 1: comparação das concentrações de metais Fonte SPC

\begin{tabular}{|l|l|l|}
\hline \multirow{3}{*}{ Metal } & Concentração na & Concentração em \\
Cobre & $0.5-2 \%$ & DSM \\
Ouro & $0.6-8 \mathrm{~g} / \mathrm{t}$ & $5-15 \%$ \\
Zinco & $5-20 \%$ & $2-20 \mathrm{~g} / \mathrm{t}$ \\
Chumbo & $5-20 \%$ & $5-50 \%$ \\
\hline & Valor Médio da & $3-23 \%$ \\
\hline & Tonelada sobre Terra & Valor Médio da \\
& Tonelada emDSM \\
\hline & US $50-200 \$$ & US $800-1500 \$$ \\
\hline
\end{tabular}

A lista de minérios comunicada acima não é exaustiva. Seria injusto não citar o cobalto, tanto esse metal é importante do ponto de vista geoestratégico para a fabricação de baterias recarregáveis, para a indústria da defesa ou para a indústria de tecnologia de ponta. ${ }^{10}$

10 PAILLARD Christophe-Alexandre. La question des minerais
Emborao DSM esteja incluído na Economia Azul unicamente graças à sua localização no mar, apesar de sua natureza potencialmente não "sustentável", nos parece legítimo expandir nossas considerações introdutórias a outras economias tais como a do conhecimento e isso por diversas razões.

Em primeiro lugar, a Economia Azul como a biomimética, a ecologia industrial e a economia circular são, em realidade, prolongamentos da economia do conhecimento como nos ensina o Professor Idriss Aberkane. ${ }^{11}$ Em segundo, a atividade de DSM pode ter um impacto negativo sobre o conhecimento quando ela destrói o meio ambiente que a contém. Por fim, com a economia do conhecimento não há mais incompatibilidade entre crescimento infinito e recursos finitos, visto que, contrariamente aos bens materiais, o conhecimento é $a$ priori infinito. ${ }^{12}$

Assim, pelo menos de um ponto de vista da criação das riquezas, a economia baseada no conhecimento foge ao paradigma trágico da finitude dos recursos evocada logo no início, o que a torna uma economia mais aconselhável.

A comparação, ou mesmo a oposição, da Economia Azul e da economia baseada no conhecimento não tem outro objetivo senão colocar o DSM em um contexto global a fim de detectar os atrativos e as consequências, o que pode parecer pertinente no contexto dessas considerações introdutórias.

De maneira judiciosa para nosso estudo sobre o DSM, o Professor Aberkane ${ }^{13}$ concentra numerosas dessas apresentações sobre a economia do conhecimento sobre a "revolução a vir" do biomimetismo. ${ }^{14} \mathrm{Em}$

stratégiques, enjeu majeur de la géoéconomie mondiale. Géoéconomie. Ruées sur les minerais stratégiques.N. ${ }^{\circ}$ 59, pág. 17-32, França, 2011. DOI: 10.3917/geoec.059.0017. Disponível em: <https://www.cairn.info/revue-geoeconomie-2011-4-page-17.htm>. Acesso em: 10 abril 2019.

11 ABERKANE Idriss J. Économie de la Connaissance. Paris: Fondation pour l'innovation politique, pág. 9, 2015. Disponível em: <http://www.fondapol.org/wp-content/uploads/2015/05/noteIdriss-AberkaneFR-WEB.pdf> . Acesso em: 20 de out. 2018.

12 ABERKANE Idriss J. Économie de la Connaissance. Paris: Fondation pour l'innovation politique, pág. 11, 2015. Disponível em: <http://www.fondapol.org/wp-content/uploads/2015/05/ note-Idriss-AberkaneFR-WEB.pdf>. Acesso em: 20 de out. 2018.

13 IDRISSABERKANE.ORG. Idriss J. Aberkane. Disponível em: $<$ https://idrissaberkane.org/index.php/category/on-en-parle/ economie-de-la-connaissance-fr/>. Acesso em: 18 out. 2018.

$14 \mathrm{O}$ biomimetismo consiste em extrair conhecimento da natureza a fim de se inspirar desta última para inovar duravelmente. Ver infra- 
suas intervenções, por exemplo, ele aborda muitas vezes espécies marinhas, como o caramujo marinho mu-conotoxina, cujo veneno a um grande valor comercia ${ }^{15}$ para as pesquisas em neurociências, ou ainda o camarão-louva-a-deus-palhaço, também conhecido como lagosta-boxeadora, que comporta em si somentediversos segredos tecnológicos. ${ }^{16}$

Além do fato desses exemplos de espécies marinhas mostrarem o interesse do enfoque que visa a apor ou opor a economia do conhecimento e a Economia Azul, a demonstração do Professor Aberkane nos convida a pesar, com a maior atenção, as escolhas a serem feitas em matéria de atividade humana em relação ao mar.

Nesse mesmo quadro de tensões entre a proteção do meio ambiente, receptáculo de conhecimento de um lado, e a resposta de necessidade em matéria-prima de outro, outros falam do "enigma", no sentido de uma escolha Corneliana, da exploração DSM. ${ }^{17}$ Numerosos estudos científicos colocam, assim, em evidência a riqueza da biodiversidade das zonas nas quais estão localizadas os diferentes depósitos polimetálicos e seus potenciais em termos de conhecimento.

É muito difícil ser exaustivo a esse respeito, entretanto, podemos nos referir a estudos essenciais como os dirigidos pela Autoridade Internacional dos Fundos Marinhos, ${ }^{18}$ os conduzidos pela Comunidade do Pacífico no âmbito do projeto «SPC-EU Deep Sea Mineral $»^{19}$ e por fim o relatório do consórcio Ecorys, MRAG,

audiência do Professor Idriss J. Aberkane no Conselho Econômico, Social e do Meio ambiente em nota de rodapé pág. 10.

15 Ver infra-audiência do Professor Idriss Jamil no Conselho Econômico, Social e do Meio ambiente em nota de rodapé pág. 10. O preço de mercado para a conotoxina oscila por volta de $800 \$$ US o grama.

16 Para mais detalhes sobre a economia do conhecimento e o valor potencial atribuído a conotoxina, acessar o vídeo da audiência do Professor Idriss J. Aberkane no Conselho Econômico, Social e do Meio ambiente, em 18 nov. 2015. Disponível em: https://www.youtube.com/watch?v=lOG96QPlitM. Acesso em: 18 out. 2018.

17 SHUKMAN, David. Renewables' deep-sea mining conundrum.BBC News. Science \& Environment, 11 de abril de 2017. 1 vídeo (1 min.). Disponível em: <https://www.bbc.com/news/ science-environment-39347620>. Acesso em 15 out. 2018.

18 INTERNATIONAL SEABED AUTHORITY (ISA). Se referir aos diversos estudos científicos do ISA como, por exemplo: Biodiversity, species ranges, and gene flow in the abyssal Pacific nodule province:predicting and managing the impacts of deep seabed mining. ISA TechnicalStudy: N. 3, 2008, 45 p. Disponível em: $<$ https://ran-s3.s3.amazonaws.com/isa.org.jm/s3fs-public/files/ documents/techstudy3.pdf>. Acesso em: 13 out. 2018.

19 COMMUNAUTÉ DU PACIFIQUE. An Assessment of the Costs and Benefits of Mining Deep-sea Minerals in the Pacific Is-
UNEP submetidos ao Parlamento Europeu em 2015. ${ }^{20}$

Se certos estudos de impacto parecem mostrar que o DSM é sujeito a provocar perturbações duráveis, ${ }^{21}$ outros minimizam esses impactos ${ }^{22}$ ou insistem sobre a carência de conhecimentos para desenvolver um julgamento. ${ }^{23}$ Podemos, no entanto, extrair desses estudos a descoberta de uma grande variedade de organismos desconhecidos adaptados à vida em condições extremas.

Para retomar a lógica da economia do conhecimento, a compreensão da adaptação desses organismos à vida extrema é suscetível de proporcionar mais ao homem do que o minério.

Contudo, é pouco provável que uma intervenção nessas profundezas não ocorra sem impacto algum para a fauna e a flora abissal e hadal ${ }^{24}$ que apresentam uma capacidade de regeneração muito lenta. ${ }^{25}$ Talvez seja por

land Region. Deep-sea Mining Cost-Benefit Analysis. Suva, Fiji: Communauté du Pacifique, 2016. Technical Report SPC00035. Disponível em: <http://dsm.gsd.spc.int/images/pdf_files/PIR_CBA_ Report.pdf $>$. Acesso em: 20 out. 2018. ISBN: 978-982-00-0955-4.

20 ECORYS AND CONSORTIUM PARTNERS. Study to investigate the state of knowledge of deep-sea mining. European Commission - DG Maritime Affairs and Fisheries. Rotterdam/Brussels, 2014. Disponível em: <https://webgate.ec.europa. $\mathrm{eu} / \mathrm{maritimeforum} / \mathrm{en} /$ file $/ 7434 /$ download?token $=\mathrm{Vms} 656 \mathrm{R} 2>$. Acesso em: 13 out. 2018.

21 AUTORITÉ INTERNATIONALE DES FONDS MARINS. Note relative à l'impact probable sur le milieu marin des activités d'exploration et d'exploitation minière des nodules. Affaire $n^{\circ} 17$ du Tribunal International du Droit de la Mer, 26 août 2010.Disponível em: <https://www.itlos.org/fileadmin/itlos/documents/cases/case_no_17/ISA_1_fr.pdf $>$

Acesso em: 15 out. 2018.

22 COMMUNAUTÉ DU PACIFIQUE. Pacific-ACP States regional environmental management framework for deep sea minerals exploration and exploitation, p. 25 e seguintes. Suva, Fiji, June 2016. ISBN: 978-982-00-1007-9.Disponível em: <http:// dsm.gsd.spc.int/images/public_files_2016/REMF2016.pdf $>$. Acesso em: 13 out. 2018.

23 GOLLNER, S.; KAISER, S.; MENZEL, L.; JONES, D. O., BROWN, A.; MESTRE, N. C., et al. (Agosto 2017). Resilience of benthic deep-sea fauna to mining activities. (Elsevier SCI Ltd, Éd.). Marine Environmental Research, Volume 129, Pág. 76-101. Disponível em: Archive Institutionnelle de l'Ifremer: <https://archimer.ifremer.fr/doc/00382/49291/49690.pdf>. Acesso em: 20 out. 2018.

24 O que é superior a uma profundidade de 6000 metros.

25 SMITH, C. R.; DRAZEN, J.; MINCKS, S. L. Deep-sea Biodiversity and Biogeography: Perspectives from the Abyss. In: INTERNATIONAL SEABED AUTHORITY SEAMOUNT BIODIVERSITY SYMPOSIUM, Honolulu, Hawaii USA, 2006. Pág. 2: « As a consequence, the biomass, growth rates, reproduction rates and recolonization rates at the abyssal seafloor are typically very low ». Disponível em: <https://www.soest.hawaii.edu/ocean- 
essa razão que foram lançadas advertências no seio da Comissão Europeia, ${ }^{26}$ do Parlamento Europeu ${ }^{27}$ e da l'IUCN, ${ }^{28}$ e no relatório do Banco Mundial supracitado.

Essas questões criaram certa polarização do debate em torno dos impactos sociais e ambientais do DSM, sobretudo com relação ao Projeto SOLWARA 1 da sociedade Nautilus ${ }^{29}$ em Papuásia-Nova Guiné. ${ }^{30}$ Por outro lado, os riscos financeiros associados ao DSM são rigorosamente apresentados nos diversos documentos de análise destinados aos investidores. ${ }^{31}$

Considerando-se o que precede, nos parece coerente apresentar o DSM quanto aos riscos e à vista disso analisar a situação em termos de escolha ou de arbitragens a efetuar entre criar riqueza através da extração mineira por um lado, e proteger o meio ambiente e terceiros

ography/mincks/publications/Smith_etal_Abyssal_biogeography_ synthesis.pdf $>$. Acesso em: 20 out. 2018.

26 SEAS AT RISK. European Commission and Azores question need for deep-sea mining, Feb. 2018. Disponível em Seas-atrisk.org: <https://seas-at-risk.org/27-deep-sea-mining/836-european-commission-and-azores-question-need-for-deep-sea-mining. html>. Acesso em: 10 março 2019.

27 SEAS AT RISK. European Parliament calls for international moratorium on deep-sea mining, January 2018. Disponível em: seas-at-risk.org/: <https://seas-at-risk.org/27deep-sea-mining/829-european-parliament-calls-for-internationalmoratorium-on-deep-sea-mining.html>. Acesso em: 13 abril 2019.

28 INTERNATIONAL UNION FOR CONSERVATION OF NATURE (IUCN). Draft mining regulations insufficient to protect the deep sea - IUCN report, 16 Jul. 2018. Disponível em : <https://www.iucn.org/: https://www.iucn.org/news/secretariat $/ 201807 /$ draft-mining-regulations-insufficient-protect-deepsea- $\%$ E2\%80\%93-iucn-report>. Acesso em: 10 março 2018.

29 NAUTILUSMINERALS.COM. Australia: Nautilus Minerals Inc. Disponível em : < http://www.nautilusminerals.com/irm/content/overview1.aspx?RID=257>. Acesso em: 22 abril 2019.

30 ROSENBAUM, H.; GREY, F. Accountability zero: a critique of Nautilus Minerals environmental and social benchmarking analysis of the Solwara 1 project. Deep Sea Mining Campaign, a project of the Ocean Foundation, 2015. Disponível em: <http:// www.deepseaminingoutofourdepth.org/wp-content/uploads/accountabilityZERO_web.pdf>. Acesso em: 18 out. 2018. Professor STEINER, Richard. Independent Review of the Environmental Impact Statement for the proposed Nautilus Minerals Solwara 1 Seabed Mining Project.Madang, Papua New Guinea, 10 Jan. 2009, 27 p.. Disponível em: <http://www.deepseaminingoutofourdepth.org/wp-content/uploads/Steiner-Independent-reviewDSM1.pdf $>$. Acesso em: 20 out. 2018.

Nautilus are you nuts!Scoop Independent News - World. 12 October 2018. Disponivel em: <http://www.scoop.co.nz/stories/ WO1810/S00078/nautilus-are-you-nuts.htm>. Acesso em: 13 março 2018.

31 NAVARRE, M.; LAMMENS, H. Opportunities of DeepSea Mining and ESG Risks.AmundiDiscussion Papers SeriesDP-24-2017. Agosto 2017, 52 p. Disponível em: <http:// researchcenter.amundi.com/>. Acesso em: 13 out. 2018. afetados por essa atividade extrativa passível de poluir, por outro lado. Contudo, a ciência mais adequada a considerar o impacto prático das escolhas parece ser a economia.

Se a análise econômica das operações de DSM escapa de toda evidência à nossa única competência jurídica, é possível, ainda assim, identificar a problemática econômica à qual pertence a opção de empreender uma atividade econômica sujeita a gerar consequências negativas sobre terceiros. Trata-se das "externalidades negativas" sobre a qual trabalhou o professor Milton Friedman. ${ }^{32}$ Essa temática de seus trabalhos é menos conhecida que seus trabalhos sobre o livre-trânsito econômico e não temos dificuldades em compreender o porquê.

O Professor Friedman, o qual certamente não se pode acusar de antiliberalismo ou de apoiar um intervencionismo forçado do Estado nas relações econômicas, reconhece que, em situações em que os atores econômicos tinham uma atividade com um impacto negativo sobre terceiros, era necessário que o Estado agisse a fim de impedir ou de reduzir esse impacto negativo.

Com vista a especificar o pensamento de Milton Friedman em relação às "externalidades negativas" e a maneira cuja a poluição deve ser considerada em relação a toda atividade econômica, é possível referir-se à citação seguinte:

"o real problema não é eliminar a poluição, mas tentar
estabelecer o equilíbrio que apresentará a boa dose de
poluição: um valor cujo ganho para a redução da poluição
será maior que a perda para o sacrifício das outras coisas
boas (...). Si formos além disso, nós sacrificaríamos mais do
que ganhamos". ${ }^{33}$

Em outras palavras, de acordo com essa concepção, uma "externalidade negativa" é aceitável a partir do mo-

32 Para uma análise mais completa sobre a origem do conceito econômico de « externalidade » e o papel deste conceito em relação às considerações ambientais, ver: GROLLEAU, Gilles; SALHI, Salima. L'externalité et la transaction environnementale : les deux faces de la même pièce? Économie rurale, 311, pp. 4-18. Maio-Junho 2009. Disponível em: $<$ http://journals.openedition.org/economierurale/880>. DOI: $<$ https://doi.org/10.4000/economierurale.880>. Acesso em: 08 abril 2019.

33 Tradução livre do inglês de CAMELO, Bradson; DUTRA DE BARROS, Juliana. Les principales solutions pour les externalités en droit de l'environnement au Brésil. Verfassung und Recht in Übersee / Law and Politics in Africa, Asia and Latin America, Vol. 44, No. 3, pp. 354-363, Germany: Ed. Nomos Verlagsgesellschaft $\mathrm{mbH}, 2011$. Disponível em : <https://www.jstor.org/stable/43240885>. Acesso em: 12 abr. 2019. 
mento que ela não ultrapassa o ganho econômico da atividade poluidora. É ainda necessário que o impacto econômico de toda poluição seja avaliado de maneira extremamente correta e exata, o que muitas vezes está longe de acontecer.

Entretanto, mesmo no tratamento miltoniano das "externalidades negativas", incumbe ao Estado prevenir e impedir o desenvolvimento de atividades que tenham um impacto extremamente negativo sobre os demais indivíduos. Resta saber se tal responsabilidade do Estado o engaja a reparar em caso de danos e qual será a distribuição dessa obrigação de maneira geral.

Visto que temos de voltar aos conceitos de obrigações, por conseguinte ao direito, existem diversos instrumentos jurídicos que permitem apreender as situações de risco e as obrigações decorrentes desses riscos a fim de fornecer determinados critérios de arbitragem no tratamento dessas problemáticas.

Em direito internacional, podemos, por exemplo, fazer referência a varias obrigaçoes o varios principios como: o "Princípio de Precaução", 34 à obrigação de impedir a poluição que resulta das operações de mineração em mar, ${ }^{35}$ à obrigação de impedir os danos transfronteiriços, ${ }^{36}$ à obrigação de preservar a biodiversidade. $^{37}$

\footnotetext{
34 CONFÉRENCE des Nations Unies sur l'environnement et le développement. Principe 15 de la Déclaration de Rio sur l'Environnement et le Développement.Vol. I. Rio de Janeiro: NATIONS UNIES, 1992. Disponível em: <https://www.un.org/ french/events/rio92/aconf15126vol1f.htm>. Acesso em: 18 out. 2018.
}

Código Mineiro da ISA ver infra, parecer do International Tribunal for the Law of the Sea, Responsibilities and obligations of States sponsoring persons and entities with respect to activities in the Area (Request for Advisory Opinion submitted to the Seabed Disputes Chamber), Case 17. Disponível em: <https://www.itlos.org/cases/list-of-cases/case-no-17/>. Acesso em: 22 out. 2018.

35 NATIONS UNIES. Convention des Nations Unies sur le droit de la mer (avec annexes, acte final et procès-verbaux de rectification de l'acte final en date des 3 mars 1986 et 26 juillet 1993). Recueil des Traités. Montego Bay, Jamaica, 16 Nov. 1994. Disponível em: $<$ https://www.un.org/depts/los/convention_agreements/texts/ unclos/unclos_f.pdf $>$. Acesso em: 22 out. 2018. Artigo 208.

36 NATIONS UNIES. Convention des Nations Unies sur le droit de la mer (Partie XII). Déclaration de Rio sur l'Environnement et le Développement. Parecer do International Tribunal for the Law of the Sea, ver supra apontamento 21.

37 Ver o Artigo 3 da Convenção sobre a Diversidade Biológica, NATIONS UNIES, 1992. Disponível em: < https://www.cbd.int/ convention/text/>. Acesso em: 18 out. 2018)
Em direito privado, podemos pensar na obrigação de um estudo de impacto ambiental para atividade suscetível de gerar danos, ${ }^{38}$ no Princípio de Poluidor Pagador, ou no Princípio de Responsabilidade Limitada quando uma atividade é julgada digna de ser apoiada, apesar do risco que ela apresenta. Essas regras têm, portanto, vocação a serem consideradas com relação ao DSM.

Suficientemente importantes para serem evocadas em linha de fundo de todo estudo sobre o DSM, essas questões não farão, entretanto, o objeto de nossa análise. Se essa abordagem parece relevante, confiaremos a outros o cuidado de estudá-la. Nesse estudo iremos nos concentrar sobre outra questão jurídica prejudicial e mais estrutural, o que tem como efeito possível acentuar os riscos associados ao DSM.

O problema jurídico sobre o qual gostaríamos de nos concentrar nesta análise é o da diferença, inelutável ou não, existente entre o regime da "Zona" submetida à Autoridade Internacional dos Fundos Marinhos (ISA) e os regimes dos fundos marinhos submetidos à jurisdição dos Estados Costeiros. Lembramos que a Convenção das Nações Unidas sobre o Direito do Mar (CDM), assinada em Montego Bay em $1982^{39}$ e o Acordo Relativo à Aplicação da Parte XI da Convenção das Nações Unidas sobre o Direito do Mar do 10 de dezembro 1982 (Acordo sobre a Parte XI), ${ }^{40}$ criaram essa dicotomia de quadro jurídico aplicável aos fundos marinhos.

Gostaríamos de mencionar, ainda, que, muito resumidamente, os Estados Costeiros exercem quer a plena soberania quer certos direitos soberanos até o limite da plataforma continental; além desse limite, é o regime dos grandes fundos marinhos decretado pela CDM e pelo Acordo sobre a Parte XI que é submetido à competência exclusiva da ISA.

Assim, observamos dois quadros regulamentares, entretanto na realidade constatamos uma multiplicidade de regimes visto que cada Estado Costeiro soberano terá suas próprias regras tendo como propósito de en-

\footnotetext{
38 NACÕES UNIDAS. Convenção das Nações Unidas sobre o Direito do Mar, Ibid, Artigo 206.

39 NACÕES UNIDAS. Convenção das Nações Unidas sobre o Direito do Mar, Ibid.

40 NACÕES UNIDAS. Acordo relativo à aplicação da Parte XI da Convenção das Nações Unidas sobre o Direito do Mar, de 10 de dezembro de 1982. New York: NACÕES UNIDAS, 28 Jul.1994. Disponível em: <https://www.admin.ch/opc/fr/classified-compilation/20040580/201110060000/0.747.305.151.pdf >. Acesso em: 18 out. 2018.
} 
quadrar o DSM. No entanto, há, sempre, um período de latência antes que um regime jurídico definido aflore para cada nova atividade humana, o que é o caso, aliás, para o DSM, mas, além disso, toda atividade de mineração requer em geral um enquadramento jurídico e institucional multidisciplinar.

Existem numerosos "contraexemplos" de enquadramentos jurídicos e institucionais inadequados que se convencionou denominar "a maldição dos recursos" O modelo norueguês ${ }^{42}$ ilustra, todavia a pertinência de um enquadramento jurídico e institucional multidisciplinar sólido para responder aos desafios levantados pelas atividades de mineração em termos de atribuição e de gerenciamento de licenças, de boa governança, de proteção do meio ambiente, de criação de autoridades independentes para controlar a aplicação das regras, para não citar que esses setores.

É forçoso constatar que, em relação ao DSM, tudo resta a ser feito. Essa observação pode ser aplicada tanto para o regime da "Zona" dos grandes fundos marinhos submetidos à ISA, que, para os regimes aplicáveis aos solos e subsolos marinhos, que são submetidos à soberania ou aos direitos soberanos dos Estados Costeiros. Assim, por exemplo,o Código Mineiro da Autoridade ainda não estáconcluído e certas submissões à ISA por investidores pioneiros tais como o Ifremer, mostram claramente que categorias inteiras do regime do DSM na Zona restam a ser definidas. ${ }^{43}$

\footnotetext{
${ }^{41}$ SACHS, Jeffrey; WARNER, Andrew. Natural resource abundance and economic growth. NBER Working Paper, No5398, 54 p., Cambridge: National Bureau of Economic Research. December 1995. DOI: 10.3386/w5398. Disponível em: <https://www.nber. org/papers/w5398.pdf>. Acesso em: 15 out. 2019.

FRANKEL Jeffrey A. The natural resource curse: a survey of diagnoses and some prescriptions. In: AREZKI Rabah; PATTILLO Catherine; QUINTYN Marc; ZHU Min, Commodity price volatility and inclusive growth in low-income countries. Washington, D.C.: International Monetary Fund, 2012. P. 7-34. CID W P 233; HKS RWP 12 - 014. Disponível em: < https://www.elibrary.imf.org/doc/ IMF071/12631-9781616353797/12631-9781616353797/Other_ formats/Source_PDF/12631-9781475544541.pdf?redirect=true>. Acesso em: 13 março 2019. Opportunities of DSM and ESG Risks, AmundiDiscussion Papers Series, Ibid infra nota 21, p. 4.

42 BORDAHANDY Pierre-Jean. The legal Status of offshore rigs. 1998. Trabalho de Conclusão de Curso (Master de quoi?) Centre de Droit Maritime et des Transports - CDMT, Faculté de Droit et des Sciences Politiques, Aix-Marseille Université, 1998.

43 INSTITUT FRANÇAIS DE RECHERCHE POUR L'EXPLOITATION DE LA MER (IFREMER). Developing a Regulatory Framework for Mineral Exploitation in the Area Submission of Ifremer. Disponível em: <https://ran-s3. s3.amazonaws.com/isa.org.jm/s3fs-public/ifremer.pdf $>$ Acesso
}

A nível dos Estados Costeiros, o problema continua a se expandir não somente em razão do número dos Estados envolvidos, mas também pela dificuldade de reproduzir de Estado em Estado as instituições e as competências necessárias à medida que os ambientes e culturas jurídicas ou outras, diferem. A zona de depósitos minerais em águas profundas, essencialmente localizada no Pacífico Sul, diversos Estados Costeiros estão suscetíveis de abrigar depósitos de minerais em sua plataforma continental. ${ }^{44}$

É nesse contexto que parece-nos estar inscrita a iniciativa do projeto SPC-EU que visa fornecer assistência aos Estados Costeiros do Pacífico para que estes enfrentem as necessidades do enquadramento jurídico e administrativo necessários ao desenvolvimento da atividade de DSM. ${ }^{45}$

Se o projeto deu efetivamente origem, entre outros, ${ }^{46}$ a um tipo de documento (ou guia) batizado RLRF ${ }^{47}$, a título de enquadramento regulamentar para os Estados Costeiros do Pacífico que desejavam desenvolver essa atividade sobre suas plataformas continentais, essa contribuição é julgada bastante severamente pelas ONG como "Blue Ocean Law" et "Pacific Network on Globalisation" em seu relatório comum sobre o DSM no Pacífico. ${ }^{48}$

em: 11 out. 2018.

44 Além da Papuásia-Nova Guiné já citada, diversos países do Pacífico Sul fizeram o levantamento de tais recursos como as Ilhas Salomão, Tonga, Fiji, Kiribati, as Ilhas Marshall, Niue, os Estados federados de Micronésia, Nauru, Palau, Samoa, Vanuatu, Niue et as Ilhas Cook.

45 PACIFIC COMMUNITY (SPC) - EU (EUROPEAN UNION). Deep Sea Minerals Project. Pacific ACP States. About The SPC-EU Deep Sea Minerals Project. Disponível em: <http://dsm. gsd.spc.int/>. Acesso em: 11 out. 2018.

46 PACIFIC COMMUNITY (SPC). Achievements of the SPC- EU Deep Sea Minerals Project. Strengthening the management of deep sea minerals in the Pacific. Nabua, Fiji Islands. March 2011-March 2014, 12 pág. Disponível em: < http://dsm.gsd. spc.int/public/files/Deep $\% 20$ Sea $\% 20$ Minerals $\% 20$ Projects $\% 20$ Achievements\%20Brochure_V2.pdf>. Acesso em: 11 out. 2018. Ver igualmente SWADDLING Alison. Pacific-ACP States - Regional environmental management framework for deep sea minerals exploration and exploitation. Prepared under the SPCEU EDF 10 Deep Sea Minerals Project by Alison SWADDLING, Environment Advisor. Suva, Fiji. June 2016. 100 p. Disponível em: <http://dsm.gsd.spc.int/images/public_files_2016/REMF2016. pdf>. Acesso em: 15 out. 2018. ISBN: 978-982-00-1007-9.

47 PACIFIC COMMUNITY (SPC). Pacific-ACP States Regional Legislative and Regulatory Framework for Deep Sea Minerals Exploration and Exploitation (RLRF). First Edition, July 2012. 70 p. Suva, Fiji. ISBN: 978-982-00-0557-0.

Disponível em: <http://dsm.gsd.spc.int/public/files/2014/RLRF2014. pdf $>$. Acesso em: 08 out. 2018.

48 BLUE OCEAN LAW \& PACIFIC NETWORK ON GLO- 
Sem julgamento de valores sobre os trabalhos supracitados, convém avaliar, por um lado, que o relatório das ONGs para as quais a União Europeia deseja, por meio do projeto SPC-EU, garantir o controle sobre os recursos dos Estados Costeiros do Pacífico, é de uma imparcialidade discutível; ${ }^{49}$ e, por outro lado, de fato o RLRF e os outros suportes fornecidos pelo projeto SPC-EU aos Estados Costeiros do Pacífico aparecem como irrisórios em comparação às diretrizes implementadas pela Noruega em relação às atividades, bem mais controladas, de exploração e operação petroleira e de gás no mar. ${ }^{50}$

Além da exigência de dispor de um enquadramento jurídico e institucional o mais completo possível, se adiciona também a necessidade de haver uma integração harmoniosa dele ao lado de outras diretrizes que tem a mesma finalidade. Se a diversidade de regimes aplicáveis a uma atividade no mar não suscita, necessariamente, um problema em si, a partir do momento que essa diversidade cria umaporta aberta ou bem um "fórum shopping" para uma zona ou um regime "mais favorável", parece haver, então, motivo para reflexão. Em resumo, o sistema da CDM é passível de ser posto em causa se não há harmonia entre o enquadramento da "Zona" e o das plataformas continentais com respeito ao DSM.

Paralelamente, o sistema de manejo dos grandes fundos (parte XI da CDM de 1982. reforçado do acordo de 1994 e do código mineiro em gestação juntamente à ISA), deve igualmente responder aos desafios de um mundo distinto do de 1982. No sistema de 1982 e de 1994, os Estados eram considerados como a origem de toda ação em matéria de DSM. Com a expansão de agentes econômicos como as multinacionais privadas às vezes capazes de ditar sua conduta aos próprios Estados

BALISATION. Resource Roulette - How Deep Sea Mining and inadequate regulatory frameworks imperil the pacific and its peoples: a report by Blue Ocean Law and The Pacific Network on Globalisation. Guam, 2016. 90 p. Disponível em: <https://cer.org. za/wp-content/uploads/2016/08/Resource-Roulette-Deep-seaMining-and-Inadequate-Regulatory-Frameworks.pdf $>$. Acesso em: 08 out. 2018.

49 Blue Ocean Law \& Pacific Network on Globalisation. Resource Roulette. Ibid, ver $\$ 4.3 \& 4.4$ p. 13.

50 NORWEGIAN PETROLEUM DIRECTORATE. Fundamental law of oil exploitation. Disponível em : <http://www.npd.no/ en/Regulations/Acts/>. Main regulations for oil exploitation. Disponível em: <http://www.npd.no/en/Regulations/Regulations/>. Guidelines on oil exploitation. Disponível em: < http://www.npd. no/en/Regulations/Guidelines/>. Acesso em: 03 março 2019. e,assim, a fortiori aos pequenos Estados do Pacífico, ${ }^{51} \mathrm{pa}-$ rece ter tido uma mudança de paradigma susceptível de colocar em perigo o conceito de patrimônio comum da humanidade como observaremos em nossas análises.

Essa introdução, mais extensa do que gostaríamos que fosse, teve por objetivo descrever, de uma parte, as tensões entre o desejo da criação de riquezas e os riscos possíveis do DSM, e de outra parte, as tensões entre as diferentes zonas decorrentes da CDM, visto que todos esses pontos participam à criação do quadro jurídico e institucional dessa atividade. Todavia, nossas análises no decorrer desse estudo serão essencialmente jurídicas, considerando-se as obrigações coronárias dos direitos de exploração atribuídos pela CDM e a harmonização recomendável dos regimes jurídicos e institucionais de uma tal exploração sob o duplo ângulo das operações da "Zona" e sobre as plataformas dos Estados Costeiros.

Esse enfoque é justificado, primeiramente, pelo nosso desejo de colocar em evidência o elo frequentemente esquecido e, portanto, crucial entre os direitos e as obrigações igualmente atribuídas pela CDM. Em segundo lugar, por nossa aspiração a formular uma crítica à uma interpretação, ao nosso entender, muito restritiva da CDM no sentido em que ela concede exclusividade ao menos de uma parte dos lucros do DSM, ao passo que os riscos permanecem integralmente compartilhados. Essa interpretação do conceito de patrimônio comum da humanidade parece-nos fiel a carta da CDM, porém, talvez, não à sua alma. Podemos, portanto, nos interrogar sobre o efeito de um conceito que, em teoria, tem como vocação beneficiar toda a humanidade e que, de fato, lhe concede, apenas, que uma parte das riquezas em troca das quais ela a faz suportar o conjunto de riscos e de problemas passíveis de surgir do DSM.

De acordo com a perspectiva supracitada, em uma primeira parte, abordaremos o patrimônio comum da humanidade como uma diretriz do código mineiro da ISA, e, em uma segunda parte, trataremos da escolha da harmonia perante a pluralidade dos regimes da Zona e das plataformas continentais.

51 GOETHALS Christophe; VINCENT Anne; WUNDERLE Marcus. Le pouvoir économique. Dossiers du CRISP, Belgique, $N^{\circ} 82,119$ p, 2013/2. ISBN : 978-2-87075-122-0. Disponível em: <https://www.cairn.info/revue-dossiers-du-crisp2013-2-page-11.htm?contenu=article > . Acesso em: 04 abril 2019. 


\section{0 "patrimônio comum da humanidade" como diretriz do código mineiro da ISA}

Como sabemos, conforme o artigo 136 da CDM, esse conceito se aplica, apenas, aos recursos dos grandes fundos marinhos da "Zona", O Artigo 137 da CDM prevê, entre outras medidas, que: "a humanidade toda, em cujo nome a Autoridade atua, é investida de todos os direitos sobre os recursos da Zona" e o Artigo 150 da CDM determina que as atividades conduzidas na zona tenham como propósito de "i) valorizar o patrimônio comum para o benefício de toda a humanidade".

Embora essas disposições tenham o mérito de serem relativamente claras quanto aos lucros a serem obtidos de uma atividade de DSM, parece que a questão está em aberto quanto à atitude a ser adotada para atividades que seriam muito perigosas e que não seriam, portanto, do interesse de toda a humanidade ou mesmo do interesse da maioria. Isso parece-nos justificar uma análise do conceito de patrimônio comum da humanidade em relação aos lucros em uma primeira parte, e em relação às responsabilidades ou danos em uma segunda parte.

\subsection{O patrimônio comum da humanidade para o benefício de todos}

No que diz respeito à atividade de DSM na "Zona", devemos recordar, em primeiro lugar, que, nos termos da CDM e do Acordo de 1994, é a Autoridade Internacional dos Fundos Marinhos (International Seabed Authority - ISA) que tem o direito exclusivo de conceder as licenças. Até o momento, há 29 licenças registradas na ISA para exploração de vários locais na "Zona" 52 . É relevante observar a presença, entre as 29 licenças registradas na ISA para a exploração da "Zona", de três licenças patrocinadas pelos Estados do Pacífico.

Trata-se, antes de tudo, da licença assinada pela empresa Marawa Research and Exploration Limited, patrocinada pelo Estado de Kiribati. Em seguida, há a companhia Tonga Offshore Mining Limited, patrocinada pelo Reino de Tonga. Por fim, temos a empresa Nauru Ocean Resources Incorporated, patrocinada pelo Estado de Nauru.

52 INTERNATIONAL SEABED AUTHORITY (ISA). Licenças. Disponível em: <https://www.isa.org.jm/deep-seabed-minerals-contractors $>$. Acessoem: 10 março 2019.
Pode-se notar que, para esses três Estados, na realidade são empresas privadas estrangeiras que estão efetivamente operando em plano de fundo.

Nesse nível, a situação de Nauru é particularmente reveladora da dinâmica que parece se desenhar com respeito ao DSM, uma vez que a empresa Nauru Ocean Resources Incorporated está de fato associada à outra empresa privada de direito canadense ${ }^{53}$, a Deep Green Resources Incorporated. ${ }^{54}$ É conveniente notar que o diretor da Deep Green Resources Incorporated, também, é o fundador da Nautilus Minerals Incorporated envolvida em projetos de DSM na plataforma continental de Papuásia-Nova Guiné, que discutiremos a seguir. ${ }^{55}$

Finalmente, vale ressaltar que o Estado de Nauru adotou uma legislação para regulamentar atividades de DSM que ele patrocina na "Zona" ${ }^{56}$ No entanto, o texto parece, simplesmente, transpor disposições que são mais apropriadas no nível da ISA que do estado patrocinador, o que afeta a lógica de todo o texto e levanta questões de compreensão. Além disso, encontramos poucos elementos, neste texto, relacionados ao gerenciamento de lucros que serão somente que uma fração do total, uma vez que uma parte irá diretamente para a empresa privada que realizou as operações e a outra para o resto da humanidade.

É inevitável constatar que a humanidade como um todo terá acesso, matematicamente falando, a, apenas, uma parcela dos lucros. Os danos, quanto a eles, serão suportados por todos, o que significa, na maioria das vezes, mais por uns do que por outros.

53 Bloomberg, Deep Green Metals Inc. / Deep Green Resources Inc. Disponível em: https://www.bloomberg.com/research/stocks/ private $/$ person. asp?personId $=27825562 \&$ privcapId $=111318879$ BLOOMBERG. Deep Green Metals Inc.

Disponível em: <https://www.bloomberg.com/profile/ company/1723846D:CN>. Acesso em: 08 março 2019.

54 Deep Green Resources Inc. Disponível em: https://deep. green/. Acesso em: 08 março 2019.

55 KOVEN Peter. Deep Green strikes deal with Glencore for undersea mining metals. Financial Post. Toronto, 15 June 2015. Disponível em: <https://business.financialpost.com/commodities/ mining/deepgreen-strikes-deal-with-glencore-for-undersea-miningmetals>. Acesso em: 10 março 2019.

56 REPUBLIC OF NAURU. International Seabed Minerals Act 2015. No. 26 of 2015. Disponível em: < http://extwprlegs1.fao.org/ docs/pdf/nau156055.pdf>. Acesso em: 11 março 2019. 


\subsection{O patrimônio comum da humanidade sob a responsabilidade de cada um}

O título desta seção é baseado na observação de que todas as entidades mencionadas na seção anterior são empresas privadas com capitalização limitada. Contudo, um princípio de responsabilidade limitada nos parece incompatível com o interesse da humanidade.

Em caso de dano, pode-se pensar que "o Estado Patrocinador"substituirá as entidades privadas para cumprir suas responsabilidades. Essa questão foi especificamente objeto de um pedido de parecer consultivo ao Tribunal do Direito do Mar em Hamburgo por parte da República de Nauru e do Reino de Tonga, os quais estavam preocupados com sua potencial responsabilidade durante o depósito de sua solicitação de licença na "Zona" supramencionada. ${ }^{57}$

A CDM não apenas prevê no Artigo 139(2) que o Estado patrocinador não é responsável por danos causados pelas empresas patrocinadas "se ele tiver tomado todas as medidas necessárias e apropriadas...", mas, mesmo que o Estado patrocinador fosse declarado responsável pelos danos, pode-se imaginar que, no caso de pequenos Estados do Pacífico com recursos modestos, tal responsabilidade permanecerá muito ilusória, visto que eles, simplesmente, não serão capazes de assumir suas obrigações. ${ }^{58}$

Portanto, podemos ser extremamente críticos somente em relação ao parecer consultivo de 2011 do Tribunal Marítimo, o qual resulta na limitação da responsabilidade do Estado patrocinador apenas para as atividades na própria “'Zona”, isto é, ocorrendo precisamente nos grandes fundos marítimos. Essa interpretação exclui o Estado patrocinador de toda responsabili-

\footnotetext{
57 INTERNATIONAL TRIBUNAL FOR THE LAW OF THE SEA. Responsibilities and obligations of States sponsoring persons and entities with respect to activities in the Area (Request for Advisory Opinion submitted to the Seabed Disputes Chamber), Case N. 17. Disponível em: <https://www.itlos.org/ cases/list-of-cases/case-no-17/>. Acesso em: 22 out. 2018.

58 FREESTONE, David. Responsibilities and Obligations of States Sponsoring Persons and Entities with Respect to Activities in the Area. American Journal of International Law, Vol. 105, Issue 4, pp. 755-760, October 2011. DOI:10.5305/amerjintelaw.105.4.0755.

Disponível em: https://www.cambridge.org/core/journals/american-journal-of-international-law/article/responsibilities-and-obligations-of-states-sponsoring-persons-and-entities-with-respect-to-activities-in-the-area/16A829C3EFE03FFD88C60C3C540C473C. Acesso em: 11 março 2019.
}

dade por danos que tenham ocorrido durante operações associadas de elevação até a superfície, de transporte em superfície ou por fim de processamento do minério.

Mesmo que se possa entender o argumento que consiste em dizer que a ISA tem jurisdição, apenas, no fundo do mar e não na coluna de água ascendente, consequentemente, essa divisão se estende automaticamente à responsabilidade do Estado Costeiro resultante do mesmo texto, parece-nos que essa postura revela um mal-entendido fundamental das operações de mineração e da legislação normalmente aplicável a elas.

Basta, por exemplo, uma pessoa leiga consultar a legislação norueguesa de exploração de petróleo para entender, imediatamente, que a única opção viável em tais circunstâncias é considerar a operação de mineração em sua globalidade, como única e indivisível. Com esse propósito, a legislação norueguesa, relativa à exploração de petróleo, tem por setor de aplicação a atividade petrolífera em sua totalidade e não apenas na área geográfica exata em que se realiza a atividade de extração, ou seja, a plataforma continental. ${ }^{59}$

Ademais, tomamos o exemplo de danos essencialmente ambientais porque eles são relativamente fáceis de detectar e identificar, visto que os exemplos de contaminações mineiras são numerosos. Danos como "perda de lucro" ou "perda de oportunidade" são muito mais difíceis de considerar, de fato e de direito.

De acordo com a dinâmica da economia do conhecimento do Professor Aberkhane, percebemos, perfeitamente, a perda que constitui a destruição de organismos dos grandes fundos marinhos ou mesmo, por ricochetes, outros organismos situados mais acima na coluna de água.

Talvez, ainda, seja difícil quantificar o valor dessa perda de conhecimento, mas é possível que ela seja bem superior em valor que em danos ambientais no sentido stricto sensu, se o conhecimento do fundo do mar for capaz de solucionar feridas da humanidade, como os acidentes cardiovasculares, o diabetes, a malária ou mesmo a AIDS. ${ }^{60}$ Admite-se que essa perda é atualmente

\footnotetext{
59 NORWEGIAN PETROLEUM DIRECTORATE. Regulations to Act relating to petroleum activities. Laid down by Royal Decree 27 June 1997. Disponível em: <http://www.npd.no/en/ Regulations/Regulations/Petroleum-activities/\#Section\%201>. Acesso em: 11 março 2019.

60 BERNSTEIN Aaron; LUDWIG David, MD, PhD. The Importance of Biodiversity to Medicine. November 19, 2008.
} 
apenas putativa, mas o limite de responsabilidade das empresas privadas e dos Estados patrocinadores é, no entanto, muito tangível.

As observações dessa subparte nos levam a crer que, in fine, somente a Autoridade, as comunidades do Pacífico ou simplesmente a humanidade como um todo responderão aos danos relacionados com o DSM. Efetivamente, portanto, é possível considerar que a verdadeira mutualização do DSM à humanidade não existe verdadeiramente em relação aos riscos e danos, o que merece ser ponderado.

Essa situação de responsabilidade limitada é agravada pelas propostas feitas pela República de Nauru à Autoridade Internacional dos Fundos Marinhos (ISA) como parte de sua abordagem para a elaboração do código de mineração e do quadro regulador para a exploração e extração DSM na "Zona". ${ }^{61}$

Nessa proposta, o governo da República de Nauru incita, por exemplo, a ISA a respeitar o frágil equilíbrio da viabilidade da exploração mineira em águas profundas, assegurando uma estrutura regulatória leve e pouco evolutiva.

Tal proposta pretende, de fato, colocar o código de mineração da ISA bem abaixo do mínimo necessário para limitar as "externalidades negativas" mencionadas na introdução, visto que se trata de considerar, apenas, o único critério comercial de viabilidade de tal exploração e não o custo das "externalidades negativas" que

JAMA. 2008;300(19): 2297-2299. DOI:10.1001/jama.2008.655. Disponível em: <https://jamanetwork.com/journals/jama/ article-abstract/182891>. Acesso em: 17 abril 2019. Ver igualmente: KIJJOA Anake; SAWANGWONG Pichan. DrugsandCosmeticsfromtheSea. Marine Drugs. Vol. 2(2), pp. 73-82, 2004. DOI:10.3390/md202073. Disponível em: <https://www.mdpi. com/1660-3397/2/2/73>. Ver: ARMSTRONG Claire W.; FOLEY Naomi S.; TINCH Rob; HOVE Sybille van den. Services from the deep: steps towards valuation of deep sea goods and services. Ecosystem Services, Vol. 2, December 2012, p. 2-13. Disponível em: <https://www.sciencedirect.com/science/article/pii/ S221204161200006X>. Acesso em 11 março 2019.

${ }^{61}$ INTERNATIONAL SEABED AUTHORITY (ISA). The International Seabed Authority releases stakeholder submissions to draft exploitation regulations. Jamaica, Kingston. 27 November 2018. Disponível em: <https://www.isa.org.jm/news/ international-seabed-authority-releases-stakeholder-submissionsdraft-exploitation-0>. Acesso em: 18 abril 2019. Ver sobretudo: ISA. Revised submission of the Government of the Republic of Nauru on the draft mining regulations. 5 p. Disponível em: $<$ https://ran-s3.s3.amazonaws.com/isa.org.jm/s3fs-public/documents/EN/Regs/2018/Comments/Nauru.pdf>. Acesso em: 11 março 2019. tal regulamentação pretende justamente regular ${ }^{62}$. No entanto, como já sugerido na introdução, a abordagem miltoniana está excessivamente longe de defender a interferência excessiva do regulador estatal e, na verdade, se trata apenas de uma posição mínima.

Consequentemente, essa proposta solicita aos redatores do código de mineração da ISA a considerar as legislações nacionais para o regime da "Zona". Isso não apenas nos parece contrário a carta da CDM, mas poderíamos ver nessa abordagem uma recuperação do poder legislativo da ISA no que diz respeito às atividades na "Zona".

Essa submissão deve ser distinguida daquela que consiste em invocar a aplicação da lei nacional do Estado patrocinador para envolver sua responsabilidade em sua ordem interna para operações do DSM na "Zona". Existe um precedente nesse nível, mesmo que não se enquadre totalmente no contexto jurídico mencionado acima, pois, como sabemos, os Estados Unidos da América não são parte da CDM nem do acordo de $1994 .{ }^{63}$

O fato de os EUA estarem fora do sistema da ISA para a exploração de DSM na "Zona" justifica a existência da competência de seus próprios tribunais estaduais em relação às atividades de seus atores econômicos em situações desprovidas de qualquer controle.

Apesar das críticas que podem ser feitas à ISA com relação à demora e ao rigor excessivo em seu trabalho de redação do código de mineração, pode-se pensar que essa abordagem cautelosa corresponde à necessidade de proteger os oceanos e a humanidade que são de responsabilidade de cada um.

Justifica-se que a ISA despenda tempo para implementar uma estrutura completa e clara para uma atividade em que todas as facetas e riscos estão longe de serem totalmente compreendidos. Nesse contexto, e, por uma questão de completude exclusivamente, a análise com-

\footnotetext{
62 Ver nos desenvolvimentos sobre o conceito "de externalidade negativa" e sobre a aplicação da abordagem miltoniana aos problemas de poluição em introdução pág. 6 .

63 CENTER FOR BIOLOGICAL DIVERSITY. Landmark Lawsuit Challenges U.S. Approval of Deep-sea Mineral Mining. New Ocean Gold Rush Could Hurt Marine Life Before Impacts Are Known. May 2015. Disponível em: < https://www. biologicaldiversity.org/news/press_releases/2015/deep-sea-mining-05-13-2015.html>. Acesso em: 22 abril 2019. Ver uma cópia da atribuição on-line : https://www.biologicaldiversity.org/ campaigns/deep-sea_mining/pdfs/Deep-seabedMiningComplaint_05-12-2015.pdf. Acesso em: 11 março 2019.
} 
parativa da legislação dos Estados da Costa do Pacífico com relação ao DSM pode, efetivamente, ser uma fonte de ensinamentos. ${ }^{64}$

Resta, no entanto, que a proposta da República de Nauru se encontra ao lado oposto de nossa argumentação que consiste em acreditar na necessidade de uma harmonização consistente da estrutura legal e regulamentar do DSM para que os Estados Costeiros adotem todas as regras promulgadas pelo ISA para suas atividades de mineração e não o contrário, como ilustraremos na segunda parte.

\section{A escolha da harmonia diante da pluralidade de regimes da "Zona" e das plataformas continentais}

A dualidade de zonas (as plataformas continentais / “a Zona”) não cria uma dualidade de regimes, mas sim uma infinidade de regimes aplicáveis às operações de DSM, uma vez que cada Estado Costeiro será obrigado a adotar uma estrutura jurídica e institucional em relação às atividades de DSM em sua plataforma continental. Mesmo que se considere que essa situação decorra, logicamente, da soberania que os Estados costeiros exercem sobre seus territórios marítimos, parece-nos que essa multiplicação de regimes não é aceitável.

Dados os riscos que a atividade de DSM representa para todos, pode parecer legítimo pensar que, se os riscos são compartilhados, os regimes o deveriam, também, ser para evitar um sistema de "forum shopping" (foro mais favorável) no qual empresas privadas organizam uma concorrência intensa dos regimes aplicáveis ao DSM, com um nivelamento inferior sistemático destes regimes em detrimento do interesse da comunidade.

Considerando-se essa questão, parece-nos importante evidenciar o problema da dualidade das zonas e da pluralidade dos regimes para frisar como uma harmonia

64 OLORUNDAMI Fayokemi. CHAPTER 10 - Developing an international legal framework for the environmental regulation of deep sea mining: a comparative analysis of the deep sea mining regulations of Papua New Guinea, the Cook Islands and the UnitedStates. Patrick Chaumette. Economic challenge and new maritime risks management: What blue growth? Challenge économique et maitrise des nouveaux risques maritimes : Quelle croissance bleue ? GOMILEX, Cap X libro 3.pmd, p.219, 27/07/2017. HAL Id: hal-01792237. Disponível em: https://hal.archives-ouvertes.fr/hal01792237/document. Acesso em: 19 abril 2019. desses regimes não alteraria em nada ou muito pouco a dualidade das zonas e a soberania dos Estados costeiros.

\subsection{Dualidade das zonas e pluralidade dos regimes}

A problemática abordada nesta segunda parte é tanto distinta da primeira como decorre inevitavelmente dela. Ela é distinta, uma vez que, nesta segunda parte, se trata de considerar as zonas sob jurisdição estatal, em suma, as plataformas continentais dos Estados do Pacífico nas quais podem ser realizadas atividades de DSM, contrariamente à primeira parte que tinha como referencial a "Zona" internacional, patrimônio comum da humanidade.

Ela decorre dela inevitavelmente, porque o que é aplicável na "Zona" terá, necessariamente, um impacto sobre as áreas sob soberania do Estado, na medida em que as duas categorias de zonas podem sofrer o risco de serem colocadas em concorrência pelas empresas de mineração. Ignorar ou minimizar esse fato seria, em nossa opinião, um erro.

É difícil fornecer uma análise abrangente da estrutura jurídica e institucional das plataformas continentais de cada país do Pacífico aplicável às atividades de DSM. No entanto, pode-se mencionar a adoção pelas Ilhas Cook de uma estrutura bastante desenvolvida nesse campo, já que não apenas a atividade de mineração, mas também as receitas dessa atividade são objeto de um tratamento legislativo. ${ }^{65}$ As Ilhas Cook também possuem uma "Seabed Minerals Authority", ${ }^{66}$ ou Autoridade de Minerais dos Fundos Marinhos, instituição ad hoc para gerenciar as atividades de DSM, e um "Seabed Minerals Commissionner"- "Comissário para os minérios dos grandes fundos marinhos", na pessoa do Senhor Paul Lynch. ${ }^{67}$

65 COOK ISLANDS. Seabed Minerals Act 2009 (amendé en 2015). Disponível em: <http://www.paclii.org/cgi-bin/sinodisp/ck/ legis $/$ num_act $/$ sma2015214/sma2015214.html?stem $=$ \&synonyms $=$ \&query $=$ seabed $\% 20$ minerals $>$. Acesso em: 11 março 2019. COOK ISLANDS. Income Tax AmendmentAct 2013 (2013 No. 18). Disponível em:<http://www.paclii.org/cgi-bin/sinodisp/ck/legis/num_ act $/$ itaa2013196/itaa2013196.html?stem $=\&$ synonyms $=$ \&query $=$ se abed $\% 20$ minerals $>$. Acesso em: 11 março 2019. COOK ISLANDS. Appropriation Act 2018. Disponível em: < http://www.paclii.org/cgibin/sinodisp/ck/legis/num_act/aa2018176/aa2018176.html?stem $=\&$ synonyms $=\&$ query $=$ seabed $\% 20$ minerals $>$. Acesso em: 11 março 2019 . 66 COOK ISLANDS SEABED MINERALS AUTHORITY. Seabed Minerals Authority. Disponível em: <https://www.seabedmineralsauthority.gov.ck/>. Acesso em: 11 março 2019.

67 LYNCH Paul E. Towards the development of a national 
Para finalizar, as Ilhas Cook adotaram um verdadeiro roteiro no que diz respeito à política de exploração e aproveitamento desses minerais. ${ }^{68}$

A abordagem das Ilhas Cook é, evidentemente, muito encorajadora e pode certamente ser considerada como um exemplo no Pacífico, embora ainda exista muito trabalho a ser desenvolvido para alcançar uma estrutura legal e institucional abrangente. Não obstante, o uso da abordagem das Ilhas Cook como referência sofre com o fato de que será difícil para outros estados como Kiribati, por exemplo, de obter o mesmo resultado pelo simples fato que os rendimentos dos dois estados não são comparáveis. Do nosso ponto de vista, é simplesmente impossível para Kiribati atingir tal nível, na medida em que eles já estão delegando a gestão de seu espaço aéreo sobre seu arquipélago para as Ilhas Fiji e, mais recentemente, ao que parece, para a Austrália. ${ }^{69}$ Nessas condições, é difícil ver como o gerenciamento tão intenso em termos de recursos como a exploração e aproveitamento das atividades de DSM poderia estar ao seu alcance.

O exemplo da República de Kiribati é relevante porque, além de ser um Estado patrocinador das atividades de DSM na "Zona", como mencionado anteriormen$\mathrm{te}^{70}$, a República de Kiribati também parece estar desenvolvendo essa atividade em sua plataforma continental, conforme indicado pela legislação adotada nesse sentido em 2017, a qual abrange tanto as operações de DSM na "Zona", quanto às operações de DSM na plataforma continental. ${ }^{71}$

regulatory framework for deep sea mining in the Cook Islands. October 2011, 31 p. Disponível em: <http://nouvelle-caledonie.ird. fr/content/download/41913/318906/.../Lynch.pdf>. Acesso em: 11 março 2019.

68 COOK ISLANDS SEABED MINERALS AUTHORITY. Cook Islands National Seabed Minerals Policy. Draft date: March 4, 2014 Version: 5, Edit: Cook Islands Seabed Minerals Authority, 27 p. Disponível em: <https://static1.squarespace.com/ static/5cca30fab2cf793ec6d94096/t/5cd08b7f0d9297780ea767 1a/1557171081757/Cook+Islands+Seabed+Minerals+Policy+. pdf $>$. Acesso em: 11 março 2019.

69 MINISTRY OF INFORMATION COMMUNICATION TRANSPORT AND TOURISM DEVELOPMENT. Kiribati signs Air Service Agreement with Australia (Hon Minister Speech), 4 de julho 2017. Disponível em: < https://www.micttd. gov.ki/press-release/kiribati-signs-air-service-agreement-australiahon-minister-speech>. Acesso em: 11 março 2019. "Kiribati is also keen to improve the management of its airspace and look into shifting the management from Fiji to Australia".

${ }_{70}$ Ver MARAWA RESEARCH AND EXPLORATION LTD.

Pág. 11. Disponível em: https://www.isa.org.jm/training/marawaresearch-and-exploration-ltd>. Acesso em: 17 abril 2019.

71 REPUBLIC OF KIRIBATI, Seabed Minerals Act 2017. Dis-
Saliente-se, no entanto, que, ao inverso das Ilhas Cook, a República de Kiribati não criou uma instituição específica encarregada de controlar ou gerenciar essa atividade, nem promulgou legislação sobre os rendimentos potenciais, o que explica nossas ressalvas anteriores.

Outro exemplo a considerar em relação às atividades de DSM na plataforma continental dos Estados do Pacífico é seguramente o mencionado anteriormente do projeto Solwara 1 da empresa Nautilus na Papuásia-Nova Guiné.

Considerado durante certo tempo como o que deveria ser a primeira exploração operacional de minério em águas profundas no mundo, o futuro desse projeto é muito incerto, na medida em que encontrou fortes resistências junto às comunidades locais, ${ }^{72}$ associações de defesa do meio ambiente ${ }^{73}$, bem como acusações de corrupção, aumentando, assim, as dificuldades jurídicas sobre a empresa Nautilus ${ }^{74}$, a qual parece estar sob a proteção da Lei das Falências. ${ }^{75}$

ponível em: <http://www.paclii.org/cgi-bin/sinodisp/ki/legis/ num_act $/$ sma2017134/sma2017134.html?stem $=\&$ synonyms $=\& q u$ ery $=$ seabed $\% 20$ minerals $>$. Acesso em: 15 maio 2019.

${ }^{72}$ BUSINESS \& HUMAN RIGHTS RESOURCE CENTRE. PNG: Concerns raised about negative impacts of seabed mining; need for consultation with local landholders. 2018. Disponível em: <https://www.business-humanrights.org/en/papua-new-guinea-concerns-raised-about-potential-negative-impactsof-seabed-mining-on-tuna-stocks-need-for-proper-consultationwith-local-landholders > . Acesso em: 18 maio 2019.

73 MCCRACKEN Colin J. Deep Sea Mining Company sued by environmentalists. Deep-Sea Mining Observer. My good planet, 12 December 2017. Disponível em: <http://dsmobserver. com/2017/12/deep-sea-mining-company-sued/>. Acesso em: 18 maio 2019. Ver igualmente: DAVIDSON Helen; DOHERTY Ben. Troubled Papua New Guinea deep-sea mine faces environmental challenge. The Guardian, 11 Dec. 2017. Disponível em: <https:// www.theguardian.com/world/2017/dec/12/troubled-papua-newguinea-deep-sea-mine-faces-environmental-challenge $>$. Acesso em: 11 março 2019.

74 DEEPSEAMININGOUTOFOURDEPTH.ORG. A project of Ocean Foundation. Nautilus' stock plummets as deep sea mining litigation proceeds. Deep Sea Mining Campaign. 17 July 2018.

Disponível em: <http://www.deepseaminingoutofourdepth.org/ nautilus-stock-plummets-as-deep-sea-mining-litigation-proceeds/>. Acesso em: 11 março 2019.

75 GRAUE Catherine. Deep sea miner on the verge of bankruptcy. ABC Radio Australia. Pacific Beat with Catherine GRAUE. 1 áudio (5min 51sec). 10 Jan 2019, 7:00 AM. Disponível em: <https:// www.abc.net.au/radio-australia/programs/pacificbeat/nautilusupdate/10704506>. Acesso em: 11 março 2019. Ver igualmente: KENNEDY Peter. Nautilus Minerals files for bankruptcy protection. Papua New Guinea Mine Watch. February 22, 2019. Disponível em: <https://ramumine.wordpress.com/2019/02/23/ nautilus-minerals-files-for-bankruptcy-protection/>. Acesso em: 11 
Aplicando a análise feita aos Estados precedentes à situação da Papuásia-Nova Guiné, observaremos, simplesmente, que o país não adotou uma legislação específica em matéria de operação de DSM. A Papuásia-Nova Guiné já possui uma forte tradição de mineração em terra e parece considerar que as leis em vigor também se destinam a regular as atividades no mar.

Assim, as atividades de DSM são cobertas pelo Mining Act de 1992, ${ }^{76}$ (Lei de Mineração de 1992), pelo Mining Safety Act of $1997^{77}$ (Lei de Segurança de Mineração de 1997) e por muitos outros regulamentos, como o Environment Act 2000 ${ }^{78}$ (Lei do Meio Ambiente de 2000). Finalmente, a mesma instituição é responsável por controlar e gerenciar as operações de mineração em terra e em águas profundas. ${ }^{79} \mathrm{~A}$ escolha de uma estrutura mínima ${ }^{80}$ e polivalente parece exigir ressalvas, considerando-se os riscos potenciais das operações de DSM já mencionadas.

De maneira geral, o fato de que, por muito tempo, a leitura da Convenção de 1982 levava a pensar que a exploração de DSM começaria principalmente na "Zona”, sob a responsabilidade da Autoridade Internacional dos Fundos Marinhos, a consideração do projeto Solwara 1,

março 2019.

76 INDEPENDENT STATE OF PAPUA NEW GUINEA. Papua New Guinea Consolidated Legislation-Mining Act 1992 No. 20 of 1992, Certified on: / /20. Disponível em: <http:// www.paclii.org/cgi-bin/sinodisp/pg/legis/consol_act/ma199281/ ma199281.html? stem $=\&$ synonyms $=\&$ query $=$ Mining $\% 20$ Act $\% 20$ of $\% 201992 />$. Acesso em: março 2019.

77 INDEPENDENT STATE OF PAPUA NEW GUINEA. Papua New Guinea Consolidated Legislation- Mining (Safety) Act 1997 No. 45 of 1977, Certified on: / /20.Disponível em: <http://www.paclii.org/cgi-bin/sinodisp/pg/legis/consol_act/ $\mathrm{ma1} 977151 / \mathrm{ma1}$ 1977151.html? $\mathrm{stem}=\&$ synonyms $=$ \&query $=$ Mini ng\%20safety $\% 20$ Act $>$. Acesso em: março 2019.

78 INDEPENDENT STATE OF PAPUA NEW GUINEA. Papua New Guinea Consolidated Legislation-Environment Act 2000No. 64 of 2000, Certified on: 19/4/2001. Disponível em: $<$ http://www.paclii.org/cgi-bin/sinodisp/pg/legis/consol_act/ ea2000159/ea2000159.html?stem $=\&$ synonyms $=\&$ query $=$ Environ ment $\% 20$ Act $\% 202000>$. Acesso em: março 2019.

79 INDEPENDENT STATE OF PAPUA NEW GUINEA. Papua New Guinea Consolidated Legislation, Mineral Resources Authority Act 2005, No. 18 of 2005, Certified on: 23/12/2005. Disponível em: <http://www.paclii.org/cgi-bin/sinodisp/pg/legis $/$ consol_act $/ \mathrm{mraa} 2005328 / \mathrm{mraa} 2005328 . \mathrm{html}$ ?stem $=$ \&synony $\mathrm{ms}=\&$ query $=$ Mining $\% 20$ Act $\% 20 \mathrm{of} \% 201992>$. Acesso em: março 2019.

80 A inadequação do enquadramento jurídico e institucional em Papuásia Nova-Guiné foi destacada em inúmeras ocasiões e, em particular, por grupos de ação civil. Disponível em: http:/ /actnowpng. org/sites/default/ files / 6.\%20Master $\% 20$ final $\% 20$ Green $\% 20$ paper1999\%20version_20Oct08.pdf. Acesso em: março 2019. entre outros ${ }^{81}$, sugerem que são, de fato, os depósitos localizados nas plataformas continentais dos Estados costeiros que serão, provavelmente, objeto das primeiras operações.

Isso nos leva a crer que, contrariamente a uma ideia preconcebida, talvez seja a existência de um enquadramento jurídico e institucional com fracos efeitos coercivos sobre as plataformas continentais que atraia investidores ao invés de atrair para a "Zona" dos fundos marinhos.

De fato, se retomarmos as diferentes submissões à ISA pelos Estados Patrocinadores, observamos a recorrência de avisos em direção a um sistema de código de mineração que seria muito restritivo. ${ }^{82}$

De modo oposto, é possível considerar que uma estrutura muito rudimentar também não protege o investidor que corre riscos de ser envolvido em numerosos processos judiciais e reduzir seus recursos, como durante o desenvolvimento do projeto Solwara 1. Portanto, podemos pensar que é justificável que a ISA despenda de certo tempo para estabelecer uma estrutura completa e clara para uma atividade que ainda está longe de ser totalmente controlada. Exclusivamente nesse contexto, a análise comparativa das legislações dos Estados costeiros do Pacífico em matéria de DSM pode efetivamente ser uma fonte de ensinamento. ${ }^{83}$

Por uma questão de imparcialidade em relação ao projeto Solwara, observaremos que:

81 ZHANG Denghua. China looking under the sea for opportunities in the Pacific. Papua New Guinea Mine Watch. 30 June 2018. Disponível em: <https://ramumine.wordpress.com/2018/07/02/ china-looking-under-the-sea-for-opportunities-in-the-pacific $/>$. Acesso em: março 2019.

82 INTERNATIONAL SEABED AUTHORITY (ISA). The International Seabed Authority Releases Stakeholder Submissions to Draft Exploitation Regulations.Jamaica: Kingston, 27 November 2018. Disponível em: <https://www.isa.org.jm/news/ international-seabed-authority-releases-stakeholder-submissionsdraft-exploitation-0>. Acesso em: março 2019.

83 OLORUNDAMI Fayokemi. CHAPTER 10 - Developing an international legal framework for the environmental regulation of deep sea mining: a comparative analysis of the deep sea mining regulations of Papua New Guinea, the Cook Islands and the UnitedStates. Patrick Chaumette. Economic challenge and new maritime risks management: What blue growth? Challenge économique et maitrise des nouveaux risques maritimes : Quelle croissance bleue ? GOMILEX, Cap X libro 3.pmd, p.219, 27/07/2017. HAL Id: hal-01792237. Disponível em: https://hal.archives-ouvertes.fr/hal01792237/document. Acesso em: 19 abril 2019. 
Por um lado, é possível que o esgotamento dos recursos da empresa Nautilus não esteja, de forma alguma, ligado às várias ações legais em andamento e, mais ainda, aos problemas entre os acionistas, alguns dos quais são provenientes de países sujeitos a restrições ou penalidades dos EUA. ${ }^{84}$

Por outro lado, mesmo considerando-se que a empresa Nautilus pode ter sido atraída pelo projeto Solwara 1 em razão da baixa profundidade do depósito de mega sulfitos no mar de Bismarck e não por qualquer característica particular da estrutura legal ou institucional, permanece que, diante das dificuldades encontradas pelo projeto, nossas observações sobre a necessidade de um sólido quadro jurídico e institucional permanecem válidas.

É nesse sentido que a extensão da aplicação do regime do código de mineração, em vias de ser elaborado pela ISA e normalmente aplicável somente à "Zona", às plataformas continentais dos Estados Costeiros nos parece uma opção a ser considerada.

\subsection{Dualidade das zonas e harmonia dos regimes}

Em termos de harmonização da lei aplicável às operações de DSM, já mencionamos o projeto Secretariat of the Pacific Community-European Union Deep Sea Minerals Project (SPC-EU DSM Project), o qual foi baseado na dupla constatação da necessidade dos Estados do Pacífico de se dotarem de uma legislação para regulamentar o DSM, por um lado, e a impossibilidade para a maioria dentre eles de obtê-la de maneira satisfatória por razões financeiras e técnicas.

No entanto, é forçoso constatar que o projeto SPC-EU não produziu os resultados esperados e que poucos Estados do Pacífico adotaram uma legislação adaptada às operações de DSM. No balanço do projeto SPC-EU, podemos considerar que o impulso de harmonização por parte da região do Pacífico não funciona e que, se harmonia houver, talvez seja partindo do regime da "Zona". Essa análise parece-nos justificada na medida em que as operações de DSM estão sujeitas a proble-

84 DEEPSEAMININGOUTOFOURDEPTH.ORG. NAUTILUS AGM: Deep Sea Mining company sinking in deep water. Deep Sean Mining Campaign. 26 August 2018.Disponívelem: <http:// www.deepseaminingoutofourdepth.org/nautilus-agm-deep-seamining-company-sinking-in-deep-water/> . Acesso em: março 2019. máticas semelhantes e que, se um regime foi construído para servir a humanidade como um todo, deveria ter uma vocação natural a ser aplicada extensivamente.

Essa tese é o ponto central de nossa demonstração e parece ser reforçada pela perspectiva negativa de uma multiplicidade de regimes de DSM, mais ou menos bem-sucedidos, competindo, ferozmente, entre si para atrair investidores internacionais em detrimento do interesse da humanidade, como já foi sugerido em nossa análise. É interessante notar que outros antes de nós aplicaram os ensinamentos do parecer consultivo do Tribunal Internacional do Direito do Mar, que trata de operações de DSM na "Zona" a situações de operações de DSM nas plataformas continentais dos Estados do Pacífico. ${ }^{85}$ Em caso de desacordo com o conteúdo da opinião, o método de prolongamento das soluções da "Zona" para as plataformas continentais parece-nos coerente.

Seria incorreto, em nossa opinião, ter como conclusão da experiência do projeto SPC-EU que os Estados do Pacífico não desejam criar um modelo de legislação aplicável a todos ou que pretendem reafirmar sua soberania por meio do exercício de sua liberdade de escolha de um sistema apropriado de origem nacional.

Nosso argumento não é, portanto, de reconsiderar sobre a dualidade de áreas decorrentes do direito internacional, nem retroceder ou restringir a soberania concedida ao Estado costeiro pelo Direito do Mar, mesmo se essa soberania nem sempre é exercida ativamente. ${ }^{86} \mathrm{~A}$ tese aqui desenvolvida baseia-se na obrigação dos Estados costeiros de estabelecer uma boa proteção dos territórios marítimos dos quais são responsáveis no exercício de sua soberania. ${ }^{87}$

85 MAKGILL Robert; LINHARES Ana. Deep Seabed Mining: Key Obligations in the Emerging Regulation of Exploration and Development in the Pacific. Routledge Handbook of Maritime Regulation and Enforcement. Routledge, London \& New York, 2016. Disponível em: <https://www.academia.edu/35562493/ Deep_Seabed_Mining_Key_Obligations_in_the_Emerging_Regulation_of_Exploration_and_Development_in_the_Pacific >. Acesso em: março 2019.

86 O exercício efetivo da sobenaria por certos microestados é frequentemente colocado. Ver: HERR Richard A. Microstate Sovereignty in the South Pacific: Is Small Practical? Contemporary Southeast Asia. Vol. 10, n. 2, pp. 182-196. ISEAS - Yusof Ishak Institute, September 1988. Disponível em: <https://www.jstor.org/ stable/25798002?seq=1\#metadata_info_tab_contents $>$ Acesso em: 03/2019.

87 CDM, ver o artigo 208 sobre a obrigação de proteção do meio ambiente, atividades de mineração, o artigo 210(5) sobre a poluição 
Com a estratégia de estender o código de mineração da ISA à exploração DSM nas plataformas continentais dos Estados Costeiros, o objetivo é, obviamente, a relativa harmonia de todos os regimes de DSM, devido às semelhanças das problemáticas que eles terão que administrar.

No entanto, a ideia é, sobretudo, evitar a aplicação da Teoria Econômica de Vantagens Comparativas de David Ricardo, a qual tem por efeito pôr em concorrência não apenas os Estados fornecedores desses minerais, mas também suas legislações, como havíamos mencionado anteriormente. A justificativa para essa escolha reside no fato de que os riscos reais ou potenciais da atividade de DSM determinam que o interesse da humanidade em termos de proteção prima antes do interesse da humanidade em termos de ganhos financeiros.

Se essa lógica se aplica à "Zona", de acordo com as disposições da CDM e do Acordo de 1994 que são relativas a ela, ela deverá, provavelmente, ser aplicada às plataformas continentais dos Estados, baseada por um lado, no "Princípio de Precaução"88 que, às vezes, é aplicável a eles e, por outro lado, no impacto que tais danos poderiam causar sobre a "Zona".

À parte o fato de ser uma interpretação muito livre do Direito Marítimo, capaz de levantar numerosas objeções absolutamente fundamentadas, parece-nos que duas dessas objeções merecem ser destacadas.

Em primeiro lugar, não há elementos nessa fase que provem que o futuro regime de mineração seja realmente uma panaceia e seja capaz de conciliar os interesses antagônicos existentes na linha de fundo de qualquer exploração mineira em águas profundas. Mostramos, por exemplo, por meio de comunicações direcionadas à ISA, o quanto as empresas de mineração e certos Estados patrocinadores temem um funcionamento complexo e lento da parte dessa autoridade. Assim, parece haver uma desconfiança do que é vivenciado como um

por despejo e o artigo e o artigo 211(5) \& (6) sobre a poluição acidental por navios. Ver igualmente as outras obrigações de proteção do meio ambiente: Art 192, 193, 194, 195, 204, 214:

88 Em uma tentativa de resumir esse princípio com valores e conteúdos jurídicos muito instáveis, podemos nos referir, entre outros documentos, ao relatório do serviço de pesquisa do Parlamento Europeu. Ver: BOURGUIGNON Didier. Le principe de précaution : Définitions, applications et gouvernance. EPRS - Service de recherche du Parlement européen,Service de recherche pour les députés. Déc. 2015 - PE 573.876. Disponível em: < http://www. europarl.europa.eu/RegData/etudes/IDAN/2015/573876/EPRS_ IDA\%282015\%29573876_FR.pdf>. Acesso em 10/mar/2019. risco de falta de consideração por parte de uma autoridade excessivamente administrativa cujo objetivo não é realmente produzir minério a um preço competitivo. No entanto, podemos moderar esse argumento observando que esse tipo de objeção decorre, sistematicamente, de qualquer esforço regulatório e que a verdadeira resposta está oculta como sempre nos detalhes do regime implementado.

Em segundo lugar, é provável que a desejável harmonização do regime a ser aplicada ao conjunto de operações de DSM, independentemente de onde estejam localizadas, pode ser provável que seja confrontada não apenas às leis do Estado Costeiro por meio do exercício de sua soberania, mas igualmente aos costumes locais, em vista da presença onipresente do pluralismo jurídico nos Estados do Pacífico.

De fato, em muitos Estados do Pacífico, há uma forte tradição costumeira que cria um verdadeiro pluralismo jurídico quando essa fonte de direito é reconhecida como tal pela Constituição, como é o caso, por exemplo, de Vanuatu ${ }^{89}$ e das Ilhas Salomão. ${ }^{90}$ Portanto, é bastante provável que, quando existe tal pluralismo jurídico, qualquer tipo de harmonização deva enfrentar um particularismo local bastante pronunciado. Provavelmente, isso levará a uma certa insegurança jurídica, pois, como sabemos, o hábito é geralmente oral e sua identificação e interpretação podem representar numerosos problemas.

Nessas circunstâncias, nosso desejo de ver a criação de um regime jurídico unificado, para regular a atividade de mineração em águas profundas, pode ser provavelmente adicionado à lista de desejos para um mundo melhor, já que há pontos obscuros entre o regime da "Zona" mais precisamente e o das outras áreas.

89 Ver os artigos 7, 30, 47, 74 e, sobretudo o artigo 95 da Constituição da República de Vanuatu. Artigo 95(3) da Constituição de Vanuatu: "Les règles coutumières continuent à produire tous leurs effets au sein du système juridique de la République de Vanuatu". Disponível em: <http://www.paclii.org/vu/legis/consol act_fr/cdlrdv397/>. Acesso em 10/mar/2019.

Ver neste sentido p.37: CORRIN Jennifer; PATERSON Don. Introduction to South Pacific Law. United Kingdom: Intersentia (4a Ed.), Jan. 2017, 440 pp. ISBN 9781780684123.

90 Ver Seções 76, 11 \& 112 da Constituição das Ilhas Salomão (1978). Disponível em: <http://www.paclii.org/sb/legis/consol_ act/c1978167/>. Acesso em 10/mar/2019. 


\section{Considerações finais}

A mineração em alto mar parece-nos fazer penetrar em um território inexplorado tanto em nível geográfico quanto científico, econômico e jurídico, pois, como vimos, as interrogações e os medos são muitos. Disso decorrem tensões quanto às funções e ao uso do oceano entre diferentes atores cujos interesses são difíceis de conciliar.

Após ter destacado o fato de que o regime jurídico de exploração dos fundos marinhos ainda está em desenvolvimento, também observamos que existem muitos desafios para sua construção e que, em certos aspectos, é possível que a legislação nacional restabeleça sua aparição em paralelo por intermediário do Estado patrocinador.

Nossa análise da situação do enquadramento jurídico em vigor nos Estados costeiros com anseios de permitir uma atividade DSM nas plataformas continentais é, provavelmente, ainda mais preocupante, pois observamos a alta probabilidade de que esses estados não estejam em condições financeiras ou técnicas de estabelecer tal quadro jurídico.

Com base nesse contexto, e, após observar o relativo fracasso do projeto SPC-EU que tinha por vocação oferecer aos Estados do Pacífico uma estrutura jurídica para as operações de DSM em suas plataformas continentais, sugerimos que tal referência ou harmonização viesse, então, do regime internacional em gestação juntamente à ISA.

Existem excelentes razões de fundo e de direito para tal abordagem, embora tenhamos sido obrigados a reconhecer que uma abordagem por extensão ou analogia será difícil nesse campo e que será necessário que cada estado aceite essa solução. No entanto, dada a conexão entre os oceanos e as possíveis consequências do DSM para a humanidade como um todo, é necessário que a abordagem que formulamos seja considerada. Além disso, parece que uma abordagem semelhante à esta sugerida neste artigo, a saber, a extensão do papel de liderança da ISA, seja adotada em relação à preservação da Biodiversidade do alto mar.

Além disso, mesmo no caso de tal harmonização ser possível, haverá muitos pontos de direito a serem esclarecidos, tanto em termos da aplicação do regime internacional aos Estados costeiros que possuem direi- to consuetudinário incompatível quanto em termos do conteúdo do regime internacional para o qual temos sérias preocupações considerando-se o parecer consultivo do tribunal de direito do mar que, em nossa opinião, é inadequada para a mineração.

Além da especificidade prática de problemas relacionados à mineração em alto mar, em geral, o DSM se inscreve, apesar disso, em problemáticas bem conhecidas do direito do mar, a saber: a necessidade de não deixar recursos na categoria "res communis" correndo o risco de vê-los desaparecer em benefício do primeiro a chegar, porque o que pertence a todos pertence, sobretudo, aos poderosos que se servirão sem contar e, ao mesmo tempo, a dificuldade de garantir que aquele a quem foram concedidos direitos sobre determinados recursos faça uma administração e um manejo adequados.

É inevitável constatar que a esperança do que pode ser chamado de terceira via, que é a da parte XI da Convenção sobre o Direito do Mar de 1982, complementada pelo acordo de 1994, pena a solucionar esse problema no que diz respeito aos recursos localizados na "Zona", tanto os objetivos do patrimônio comum da humanidade parecem fáceis a contornar. Paralelamente, a concessão de certos recursos ao Estado Costeiro, com base na esperança de gerenciamento e administração responsáveis por este último, parece estar sujeita à mesma dedicação.

Demonstrou-se que, no caso do DSM no Pacífico Sul, são mais ou menos as mesmas forças que estão trabalhando. A Convenção de 1982 foi redigida numa época em que as empresas não tinham completamente o mesmo poder que hoje em dia, é um fato. Conceitualmente, no entanto, o risco de monopolizar recursos permanece inalterado. Para enfrentar esse desafio, é importante focar nas condições associadas pela CDM ao compartilhamento dos recursos dos fundos marinhos com a alocação daqueles localizados nas plataformas continentais dos Estados costeiros.

Diante de recursos finitos e um meio ambiente frágil, é realmente importante que cada um assuma suas responsabilidades, mesmo quando isso consiste em admitir não ser capaz de cumprir as obrigações impostas pela CDM, para que todos se beneficiem de mais humanidade. Então, talvez, teremos finalmente algo além de martelos para ofereceroutra coisa que pregos. 


\section{Referências}

ABERKANE Idriss. Économie de la Connaissance. Paris: Fondation pour l'innovation politique, pág. 9, 2015. Disponível em: <http://www.fondapol.org/wpcontent/uploads/2015/05/note-Idriss-AberkaneFRWEB.pdf>. Acesso em: 20 de out. 2018.

ARMSTRONG Claire W.; FOLEY Naomi S.; TINCH Rob; HOVE Sybille van den. Services from the deep: steps towards valuation of deep sea goods and services. Ecosystem Services, Vol. 2, December 2012, p. 2-13. Disponível em: <https://www.sciencedirect.com/ science/article/pii/S221204161200006X>. Acesso em 11 março 2019.

AUTORITÉ INTERNATIONALE DES FONDS MARINS. Note relative à l'impact probable sur le milieu marin des activités d'exploration et d'exploitation minière des nodules. Affaire $\mathrm{n}^{\circ} 17 \mathrm{du}$ Tribunal International du Droit de la Mer, 26 août 2010. Disponível em: <https://www.itlos.org/fileadmin/ itlos/documents/cases/case_no_17/ISA_1_fr.pdf $>$ Acesso em: 15 out. 2018.

BERNSTEIN Aaron; LUDWIG David, MD, PhD. The Importance of Biodiversity to Medicine. November 19, 2008. JAMA. 2008;300(19): $2297-$ 2299. DOI:10.1001/jama.2008.655. Disponível em: $<$ https://jamanetwork.com/journals/jama/article-abstract/182891>. Acesso em: 17 abril 2019.

BLUE OCEAN LAW \& PACIFIC NETWORK ON GLOBALISATION. Resource Roulette - How Deep Sea Mining and inadequate regulatory frameworks imperil the pacific and its peoples : a report by Blue Ocean Law and The Pacific Network on Globalisation. Guam, 2016. 90 p. Disponível em: < https://cer.org.za/wpcontent/uploads/2016/08/Resource-Roulette-Deepsea-Mining-and-Inadequate-Regulatory-Frameworks. pdf>. Acesso em:

BLUE OCEAN LAW \& PACIFIC NETWORK ON GLOBALISATION. Resource Roulette. Ibid, ver \4.3\& 4.4 pág. 13.

BLOOMBERG. Deep Green Metals Inc. Disponível em: <https://www.bloomberg.com/profile/ company/1723846D:CN>. Acesso em: 08 março 2019.
BORDAHANDY Pierre-Jean. The legal Status of offshore rigs. 1998. 84 pag. Master Thesis - Direito Maritimo - Centre de droit maritime et des transports - CDMT, Faculté de Droit et des Sciences Politiques, Aix-Marseille Université, 1998.

BOURGUIGNON Didier. Le principe de précaution:Définitions, applications et gouvernance. EPRS - Service de recherche du Parlement européen,Service de recherche pour les députés. Décembre 2015 - PE 573.876. Disponível em: <http://www.europarl.europa.eu/RegData/etudes/IDAN/2015/573876/EPRS_ IDA $\% 282015 \% 29573876 \_F R . p d f>$. Acesso em: 10 março 2019.

BUSINESS \& HUMAN RIGHTS RESOURCE CENTRE. PNG: Concerns raised about negative impacts of seabed mining; need for consultation with local landholders. 2018. Disponível em: <https:// www.business-humanrights.org/en/papua-new-guineaconcerns-raised-about-potential-negative-impacts-ofseabed-mining-on-tuna-stocks-need-for-proper-consultation-with-local-landholders $>$. Acesso em: 18 maio 2019.

CAMELO, Bradson; DUTRA DE BARROS, Juliana (tradução livre de). Verfassung und Recht in Übersee / Law and Politics in Africa, Asia and Latin America, Vol. 44, No. 3, pp. 354-363, Germany: Ed. Nomos Verlagsgesellschaft mbH, 2011. Disponível em : $<$ https://www.jstor.org/stable/43240885>. Acesso em: 12 abril 2019.

CENTER FOR BIOLOGICAL DIVERSITY. Landmark Lawsuit Challenges U.S. Approval of Deep-sea Mineral Mining. New Ocean Gold Rush Could Hurt Marine Life Before Impacts Are Known. May 2015. Disponível em: <https://www. biologicaldiversity.org/news/press_releases/2015/deep-sea-mining-05-13-2015.html>. Acesso em: 22 abril 2019.

COMMISSION EUROPÉENNE. The 2018 Annual Economic Report on Blue Economy. Direction Générale des affaires maritimes et de la pêche. Vol. 01, Catálogo KL-AR-18-001-EN-N, 2018. ISBN 978-9279-81757-1, ISSN 2599-6584. DOI 10.2771/305342. Disponível em: <https://publications.europa.eu/en/ publication-detail/-/publication/79299d10-8a35-11e8ac6a-01aa75ed71a1>. Acesso em: 15 out. 2018. 
COMMUNAUTÉ DU PACIFIQUE. An Assessment of the Costs and Benefits of Mining Deep-sea Minerals in the Pacific Island Region. Deep-sea Mining CostBenefit Analysis. Suva, Fiji: Communauté du Pacifique, 2016. Disponível em: <http://dsm.gsd.spc.int/ images/pdf_files/PIR_CBA_Report.pdf $>$. Acesso em: 20 out. 2018. ISBN: 978-982-00-0955-4.

COMMUNAUTÉ DU PACIFIQUE. Pacific-ACP States Regional environmental management framework for deep sea minerals exploration and exploitation, pág. 25 e seguintes. Suva, Fiji, June 2016. Disponível em: <http://dsm.gsd.spc.int/images/public_files_2016/REMF2016.pdf > . Acesso em: 13 out. 2018. ISBN: 978-982-00-1007-9.

COMMUNAUTE DU PACIFIQUE. Pacific-ACP States. Regional environmental management framework for deep sea minerals exploration and exploitation, pág. 25 e seguintes. Suva, Fiji, 2016. Disponível em: <http://dsm.gsd.spc.int/images/public_ files_2016/REMF2016.pdf>. Acesso em: 13 out. 2018. ISBN: 978-982-00-1007-9.

CONFÉRENCE des Nations Unies sur l'environnement et le développement. Principe 15 de la Déclaration de Rio sur l'Environnement et le Développement.Vol. I. Rio de Janeiro: NATIONS UNIES, 1992. Disponível em: <https://www.un.org/french/events/rio92/ aconf15126vol1f.htm>. Acesso em: 17 out. 2018. Código Mineiro da ISA ver infra, parecer do International Tribunal for the Law of the Sea, Responsibilities and obligations of States sponsoring persons and entities with respect to activities in the Area (Request for Advisory Opinion submitted to the Seabed Disputes Chamber), Case 17. Disponível em: <https://www. itlos.org/cases/list-of-cases/case-no-17/>. Acesso em: 22 out. 2018.

CONSEIL ECONOMIQUE, SOCIAL E ENVIRONNEMENTAL. Audition d'Idriss ABERKANE sur 1 'économie de la connaissance et le biomimétisme, 18 Nov. 2015. 1 vídeo (28m44s). Disponível em: IDRISSABERKANE.ORG: < https://idrissaberkane.org/index.php/2015/11/18/leconomie-de-la-connaissanceet-le-biomimetisme/>. Acesso em: 16 abril 2018.

COOK ISLANDS. Appropriation Act 2018. Disponível em: <http://www.paclii.org/cgi-bin/sinodisp/ck/legis/num_act/aa2018176/aa2018176.html?ste $\mathrm{m}=\&$ synonyms $=\&$ query $=$ seabed $\% 20$ minerals $>$. Acesso em: 11 março 2019.
COOK ISLANDS. Income Tax Amendment Act 2013 (2013 No. 18). Disponível em: $<$ http://www. paclii.org/cgi-bin/sinodisp/ck/legis/num_act/ itaa2013196/itaa2013196.html?stem $=$ \&synonyms $=$ \& query $=$ seabed $\% 20$ minerals $>$. Acesso em: 11 março 2019.

COOK ISLANDS. Seabed Minerals Act 2009 (amendé en 2015).Disponível em: <http://www.paclii.org/ cgi-bin/sinodisp/ck/legis/num_act/sma2015214/ sma2015214.html? stem $=\&$ synonyms $=\&$ query $=$ seab ed\%20minerals>. Acesso em: 11 março 2019.

COOK ISLANDS SEABED MINERALS AUTHORITY. Seabed Minerals Authority. Disponível em: <https://www.seabedmineralsauthority.gov.ck/>. Acesso em: 11 março 2019.

COOK ISLANDS SEABED MINERALS AUTHORITY. Cook Islands National Seabed Minerals Policy. Draft date: March 4, 2014 Version: 5, Edit: Cook Islands Seabed Minerals Authority, 27 p. Disponível em: <https://static1.squarespace.com/ static/5cca30fab2cf793ec6d94096/t/5cd08b7f0d9297 780ea7671a/1557171081757/Cook+Islands+Seabed+ Minerals+Policy+.pdf>. Acesso em: 11 março 2019.

CORRIN Jennifer; PATERSON Don. Introduction to South Pacific Law. United Kingdom: Intersentia (4a Ed.), Jan. 2017, 440 pp. ISBN 9781780684123.

DAVIDSON Helen; DOHERTY Ben. Troubled Papua New Guinea deep-sea mine faces environmental challenge. The Guardian, 11 Dec. 2017. Disponível em: <https://www.theguardian.com/world/2017/dec/12/ troubled-papua-new-guinea-deep-sea-mine-faces-environmental-challenge>. Acesso em: 11 março 2019.

DEEPSEAMININGOUTOFOURDEPTH.ORG. A project of Ocean Foundation. Nautilus' stock plummets as deep sea mining litigation proceeds. Deep Sea Mining Campaign. 17 July 2018. Disponível em: <http://www.deepseaminingoutofourdepth.org/ nautilus-stock-plummets-as-deep-sea-mining-litigationproceeds/>. Acesso em: 11 março 2019.

DEEPSEAMININGOUTOFOURDEPTH.ORG. NAUTILUS AGM: Deep Sea Mining company sinking in deep water. Deep Sean Mining Campaign. 26 August 2018.Disponível em: <http://www.deepseaminingoutofourdepth.org/nautilus-agm-deep-seamining-company-sinking-in-deep-water/>. Acesso em: 11 março 2019. 
DEEPGREEN RESOURCES INC. Disponível em: https://deep.green/. Acesso em: 08 março 2019.

ECORYS AND CONSORTIUM PARTNERS. Study to investigate the state of knowledge of deep-sea mining. European Commission - DG Maritime Affairs and Fisheries. Rotterdam/Brussels, 2014. Disponível em: <https://webgate.ec.europa.eu/maritimeforum/ en/file/7434/download?token $=$ Vms656R2 $>$. Acesso em: 13 out. 2018.

FRANKEL Jeffrey A. The natural resource curse: a survey of diagnoses and some prescriptions. In: AREZKI Rabah; PATTILLO Catherine; QUINTYN Marc; ZHU Min, Commodity price volatility and inclusive growth in low-income countries. Washington, D.C.: International Monetary Fund, 2012. P. 7-34. W P 233; HKS RWP 12 - 014. Disponível em: <https:/ /www.elibrary. imf.org/doc/IMF071/12631-9781616353797/126319781616353797/Other_formats/Source_PDF/126319781475544541.pdf? redirect $=$ true $>$. Acesso em: 13 março 2019.

FREESTONE, David. Responsibilities and Obligations of States Sponsoring Persons and Entities with Respect to Activities in the Area. American Journal of International Law, Vol. 105, Issue 4, pp. 755-760, October 2011. DOI:10.5305/amerjintelaw.105.4.0755. Disponível em: https://www.cambridge.org/core/ journals/american-journal-of-international-law/article/responsibilities-and-obligations-of-states-sponsoring-persons-and-entities-with-respect-to-activities-inthe-area/16A829C3EFE03FFD88C60C3C540C473C. Acesso em: 11 março 2019.

GOETHALS Christophe; VINCENT Anne; WUNDERLE Marcus. Le pouvoir économique. Dossiers du CRISP, Belgique,Nº2, 119 p, 2013/2. Disponível em: < https://www.cairn.info/revue-dossiers-du-crisp2013-2-page-11.htm?contenu $=$ article $>$. Acesso em: 04 abril 2019. ISBN : 978-2-87075-122-0.

GOLLNER, S.; KAISER, S.; MENZEL, L.; JONES, D. O., BROWN, A.; MESTRE, N. C., et al. (Agosto 2017). Resilience of benthic deep-sea fauna to mining activities. (Elsevier SCI Ltd, Éd.). Marine Environmental Research, Volume 129, Pág. 76-101. Disponível em: Archive Institutionnelle de l'Ifremer: <https://archimer.ifremer.fr/doc/00382/49291/49690.pdf>. Acesso em: 20 out. 2018.
GRAUE Catherine. Deep sea miner on the verge of bankruptcy. ABC Radio Australia. Pacific Beat with Catherine GRAUE. 1 áudio (5min 51sec). 10 Jan 2019. Disponível em: <https://www.abc.net.au/radio-australia/programs/pacificbeat/nautilus-update/10704506>. Acesso em: 17 abril 2019.

GROLLEAU, Gilles et SALHI, Salima. L'externalité et la transaction environnementale : les deux faces de la même pièce? Économie rurale, 311, 4-18. MaioJunho 2009. Disponível em: $<$ http://journals.openedition.org/economierurale/880>. DOI: $<$ https://doi. org/10.4000/economierurale.880>. Acesso em: 08 abril 2019 .

HERR Richard A. Microstate Sovereignty in the South Pacific: Is Small Practical? Contemporary Southeast Asia. Vol. 10, n. 2, pp. 182-196. ISEAS - YusofIshak Institute, September 1988.

Disponível em: <https://www.jstor.org/ stable $/ 25798002$ ?seq=1\#metadata_info_tab_contents>. Acesso em: março 2019.

IDRISSABERKANE.ORG. Idriss J. Aberkane. Disponível em: <https://idrissaberkane.org/index.php/ category/on-en-parle/economie-de-la-connaissancefr/ > . Acesso em: 18 out. 2018.

INDEPENDENT STATE OF PAPUA NEW GUINEA.Papua New Guinea Consolidated Legislation-Mining Act 1992 No. 20 of 1992, Certified on: / /20. Disponível em: <http://www.paclii.org/pg/legis/consol_act/ma199281/>. Acesso em: março 2019.

INDEPENDENT STATE OF PAPUA NEW GUINEA.Papua New Guinea Consolidated LegislationMining (Safety) Act 1997 No. 45 of 1977, Certified on: / /20.Disponível em: <http://www.paclii.org/ cgi-bin/sinodisp/pg/legis/consol_act/ma1977151/ ma1977151.html?stem $=\&$ synonyms $=\&$ query $=$ Mini ng\%20safety\%20Act>. Acesso em: março 2019.

INDEPENDENT STATE OF PAPUA NEW GUINEA. Papua New Guinea Consolidated Legislation-Environment Act 2000No. 64 of 2000, Certified on: 19/4/2001. Disponível em: <http://www. paclii.org/cgi-bin/sinodisp/pg/legis/consol_act/ ea2000159/ea2000159.html?stem $=\&$ synonyms $=$ \&quer $\mathrm{y}=$ Environment $\% 20$ Act $\% 202000>$. Acesso em: março 2019. 
INDEPENDENT STATE OF PAPUA NEW GUINEA. Papua New Guinea Consolidated Legislation, Mineral Resources Authority Act 2005, No. 18 of 2005, Certified on: 23/12/2005. Disponível em: <http://www.paclii.org/cgi-bin/sinodisp/pg/legis/ consol_act $/ \mathrm{mraa} 2005328 / \mathrm{mraa} 2005328 . \mathrm{html}$ ?stem $=\&$ synonyms $=\&$ query $=$ Mining $\% 20$ Act $\% 20$ of $\% 201992>$. Acesso em: março 2019.

INSTITUT FRANÇAIS DE RECHERCHE POUR L'EXPLOITATION DE LA MER (IFREMER). Developing a Regulatory Framework for Mineral Exploitation in the Area - Submission of Ifremer.18 pp. Disponível em: <https://ran-s3.s3.amazonaws. com/isa.org.jm/s3fs-public/ifremer.pdf $>$ Acesso em: 11 out. 2018.

INTERNATIONAL SEABED AUTHORITY (ISA). Licenças. Disponível em: <https://www.isa.org.jm/ deep-seabed-minerals-contractors $>$. Acesso em: 10 março 2019.

INTERNATIONAL SEABED AUTHORITY (ISA). Biodiversity, species ranges, and gene flow in the abyssal Pacific nodule province:predicting and managing the impacts of deep seabed mining. ISA Technical Study: N. 3, 45 pág., 2008. Disponível em: <https://ran-s3. s3.amazonaws.com/isa.org.jm/s3fs-public/files/documents/techstudy3.pdf>. Acesso em: 13 out. 2018.

INTERNATIONAL SEABED AUTHORITY (ISA). Report on Developing a Regulatory Framework for Mineral Exploitation in the Area. Kingston: International Seabed Authority, March 2015. Disponível em: $<$ https://ran-s3.s3.amazonaws.com/isa.org.jm/s3fspublic/documents/EN/Survey/Report-2015.pdf>. Acesso em: 13 março 2019.

INTERNATIONAL SEABED AUTHORITY (ISA). Revised submission of the Government of the Republic of Nauru on the draft mining regulations. 5 p. Disponível em: < https://ran-s3.s3.amazonaws.com/ isa.org.jm/s3fs-public/documents/EN/Regs/2018/ Comments/Nauru.pdf $>$. Acesso em: 11 março 2019.

INTERNATIONAL TRIBUNAL FOR THE LAW OF THE SEA. Responsibilities and obligations of States sponsoring persons and entities with respect to activities in the Area (Request for Advisory Opinion submitted to the Seabed Disputes Chamber), Case N. 17. Disponível em: < https://www.itlos.org/cases/ list-of-cases/case-no-17/>. Acesso em: 22 out. 2018.
INTERNATIONAL SEABED AUTHORITY (ISA). The International Seabed Authority Releases Stakeholder Submissions to Draft Exploitation Regulations.Jamaica: Kingston, 27 November 2018. Disponível em: <https://www.isa.org.jm/news/international-seabed-authority-releases-stakeholder-submissions-draft-exploitation-0>. Acesso em: março 2019.

INTERNATIONAL UNION FOR CONSERVATION OF NATURE (IUCN). Draft mining regulations insufficient to protect the deep sea - IUCN report, 16 Jul. 2018. Disponível em : <https://www. iucn.org/: https://www.iucn.org/news/secretariat $/ 201807 /$ draft-mining-regulations-insufficient-protect-deep-sea- $\%$ E2\%80\%93-iucn-report>. Acesso em: 10 março 2018.

KENNEDY Peter. Nautilus Minerals files for bankruptcy protection. Papua New Guinea Mine Watch. February 22, 2019. Disponível em: <https:// ramumine.wordpress.com/2019/02/23/nautilus-minerals-files-for-bankruptcy-protection/>. Acesso em: 11 março 2019.

KIJJOA Anake; SAWANGWONG Pichan. Drugs and Cosmetics from the Sea. Marine Drugs. Vol. 2(2), pp. 73-82, 2004. DOI:10.3390/md202073. Disponível em: $<$ https://www.mdpi.com/1660-3397/2/2/73>. Acesso em: 13 março 2019.

KOVEN Peter. Deep Green strikes deal with Glencore for undersea mining metals. Financial Post. Toronto, 15 June 2015. Disponível em: < https://business.financialpost.com/commodities/mining/deepgreen-strikes-deal-with-glencore-for-undersea-mining-metals $>$. Acesso em: 10 março 2019.

LYNCH Paul E. Towards the development of a national regulatory framework for deep sea mining in the Cook Islands. October 2011, 31 p. Disponível em: $<$ http:// nouvelle-caledonie.ird.fr/content/download/41913/318906/.../Lynch.pdf>. Acesso em: 11 março 2019.

MCCRACKEN Colin J. Deep Sea Mining Company sued by environmentalists. Deep-sea mining Observer. My good planet, 12 December 2017. Disponível em: < http://dsmobserver.com/2017/12/deep-sea-mining-company-sued/> . Acesso em: 18 maio 2019. 
MAKGILLRobert; LINHARES Ana. Deep Seabed Mining: Key Obligations in the Emerging Regulation of Exploration and Development in the Pacific. Routledge Handbook of Maritime Regulation and Enforcement. Routledge, London \& New York, 2016. Disponível em: <https://www.academia.edu/35562493/ Deep_Seabed_Mining_Key_Obligations_in_the_ Emerging_Regulation_of_Exploration_and_Development_in_the_Pacific $>$. Acesso em: março 2019.

MARAWA RESEARCH AND EXPLORATION LTD. Pág. 11. Disponível em: https://www.isa.org.jm/ training/marawa-research-and-exploration-ltd>. Acesso em: 17 abril 2019.

MEADOWS, D. H.; MEADOWS, D.; Jørgen, R.; \& BEHRENS III, W. W. The Limits to Growth. The Club of Rome, A Report for the Club of Rome's Project on the Predicament of Mankind. New York: Universe Books, 1972. ISBN 0876631650. Disponível em: <http://www.donellameadows.org/wp-content/ userfiles/Limits-to-Growth-digital-scan-version.pdf > . Acesso em: 15 out. 2018.

MEADOWS, D. A brief and incomplete history of operational gaming in system dynamics.. System Dynamics Review, 23 (Issue 2-3), 199-203, Ed. J. D. Sterman, 2007.Disponível em: <https://www.systemdynamics.org/assets/docs/sd_games_history.pdf $>$. Acesso em: 18 out. 2018.

MINISTRY OF INFORMATION COMMUNICATION TRANSPORT AND TOURISM DEVELOPMENT. Kiribati signs Air Service Agreement with Australia (Hon Minister Speech), 4 de julho 2017. Disponível em: <https://www.micttd.gov.ki/ press-release/kiribati-signs-air-service-agreementaustralia-hon-minister-speech $>$. Acesso em: 11 março 2019.

NAVARRE, M.; LAMMENS, H. Opportunities of Deep-Sea Mining and ESG Risks.AmundiDiscussion Papers SeriesDP-24-2017, 52 pág. Agosto 2017. Disponível em: <http://research-center.amundi.com/>. Acesso em: 13 out. 2018.

NATIONS UNIES. Multilatéral - Convention des Nations Unies sur le droit de la mer (avec annexes, acte final et procès-verbaux de rectification de l'acte final en date des 3 mars 1986 et 26 juillet 1993). Conclue à Montego Bay le 10 décembre 1982. Recueil des Traités. Montego Bay, Jamaique, 16 Nov. 1994 (Registered ex officio). Disponível em: <https://www.un.org/depts/ los/convention_agreements/texts/unclos/unclos_f. pdf>. Acesso em: 22 out. 2018. Artigo 208.

NATION UNIES. Convenção sobre a Diversidade Biológica. 1992.30 p. Disponível em: <https://www. cbd.int/convention/text/>. Acesso em: 18 out. 2018.

NATIONS UNIES. Accord relatif à l'application de la partie XI de la Convention des Nations Unies sur le droit de la mer du 10 décembre 1982. Conclu à New York le 28 juillet 1994. Disponível em: $<$ https://www.admin.ch/opc/fr/classified-compilation/20040580/201110060000/0.747.305.151.pdf>. Acesso em: 18 out. 2018.

NAUTILUSMINERALS.COM. Australia: Nautilus Minerals Inc. Disponível em : < http:/ / www.nautilusminerals.com/IRM/content/default.aspx $>$. Acesso em: 22 abril 2019 .

NORWEGIAN PETROLEUM DIRECTORATE. Regulations to Act relating to petroleum activities. Laid down by Royal Decree 27 June 1997. Disponível em: $\quad<$ http://www.npd.no/en/Regulations/Regulations/Petroleum-activities/\#Section\%201>. Acesso em: 11 março 2019.

NORWEGIAN PETROLEUM DIRECTORATE. Lois fondamentales sur l'exploitation pétrolière. Disponível em : <http://www.npd.no/en/Regulations/Acts/>. Acesso em: 03 março 2019

NORWEGIAN PETROLEUM DIRECTORATE. Principaux règlements concernant l'exploitation pétrolière en mer. Disponível em : <http://www. npd.no/en/Regulations/Regulations/>. Acesso em: 03 março 2019

\section{NORWEGIAN PETROLEUM DIRECTORA-} TE. Recommandations concernant l'exploitation pétrolière en mer. Disponível em < http://www.npd. no/en/Regulations/Guidelines/>. Acesso em: 03 março 2019.

Act 29 November 1996 No. 72 relating to petroleum activities. Disponível em: < https://www.npd.no/en/regulations/acts/act-29-november-1996-no2.-72-relatingto-petroleum-activities/>. Acesso em: 15 março 2019.

Regulations to Act relating to petroleum activities.Disponível em: < https://www.npd.no/en/regulations/regulations/petroleum-activities/>. Acesso em: 15 março 2019. 
OPPORTUNITIES of Deep-Sea Mining and ESG Risks. AmundiDiscussion Papers Series.

OLORUNDAMI Fayokemi. CHAPTER 10 - Developing an international legal framework for the environmental regulation of deep sea mining: a comparative analysis of the deep sea mining regulations of Papua New Guinea, the Cook Islands and the United-States. Patrick Chaumette. Economic challenge and new maritime risks management: What blue growth? Challenge économique et maîtrise des nouveaux risques maritimes : Quelle croissance bleue ?GOMILEX, Cap X libro 3.pmd, p.219, 27/07/2017. HAL Id: hal01792237. Disponível em: https://hal.archives-ouvertes.fr/hal-01792237/document. Acesso em: 19 abril 2019.

PACIFIC COMMUNITY (SPC). Achievements of the SPC- EU Deep Sea Minerals Project. Strengthening the management of deep sea minerals in the Pacific. Nabua, Fiji Islands. March 2011-March 2014, 12 pág. Disponível em: <http://dsm.gsd.spc.int/public/files/Deep $\% 20$ Sea $\% 20$ Minerals $\% 20$ Projects $\% 20$ Achievements $\% 20$ Brochure_V2.pdf $>$. Acesso em: 11 out. 2018.

PACIFIC COMMUNITY (SPC). Pacific-ACP States Regional Legislative and Regulatory Framework for Deep Sea Minerals Exploration and Exploitation.First Edition, July 2012. 70 p. Suva, Fiji. ISBN: 978-982-00-0557-0. Disponívelem: <http://dsm.gsd. spc.int/public/files/2014/RLRF2014.pdf>. Acesso em: 08 out. 2018.

PAILLARD Christophe-Alexandre. La question des minerais stratégiques, enjeu majeur de la géoéconomie mondiale. Géoéconomie. Ruées sur les minerais stratégiques. $\mathrm{N}^{\circ}$ 59, pág. 17-32, França, 2011. Disponível em: <https://www.cairn.info/revue-geoeconomie-2011-4-page-17.htm>. Acesso em: 10 abril 2019. DOI: 10.3917 /geoec.059.0017

PAULI Gunter. The Blue Economy: 10 Years, 100 Innovations, 100 Million Jobs. The Club of Rome. Taos, New Mexico USA: Paradigm Publications, 2010. ISBN 978-0-912111-90-2.

REPUBLIC OF KIRIBATI, Seabed Minerals Act 2017. Disponívelem: < http://www.paclii.org/cgi-bin/ sinodisp/ki/legis/num_act/sma2017134/sma2017134. html? stem $=\&$ synonyms $=$ \&query $=$ seabed $\% 20$ minerals>. Acesso em: 15 maio 2019.
REPUBLIC OF NAURU. International Seabed Minerals Act 2015. No. 26 of 2015. Disponível em: $<$ http://extwprlegs1.fao.org/docs/pdf/nau156055. pdf>. Acesso em: 11 março 2019.

RÉPUBLIQUE DE VANUATU. Constitution de La République de Vanuatu (1980). Édition consolidée 2006. Entrée en vigueur, le 30 juillet 1980. Hormis les articles 87 et 93: entrés en vigueur, le 23 octobre 1979. Disponível em: <http://www.paclii.org/vu/legis/consol_act_fr/cdlrdv397/>. Acesso em: 10 março 2019.

ROSENBAUM, H.; GREY, F. Accountability zero: a critique of Nautilus Minerals environmental and social benchmarking analysis of the Solwara 1 project. Deep Sea Mining Campaign, a project of the Ocean Foundation, 2015. Disponível em: < http:// www.deepseaminingoutofourdepth.org/wp-content/ uploads/accountabilityZERO_web.pdf $>$. Acesso em: 18 out. 2018.

SACHS, Jeffrey; WARNER, Andrew. Natural resource abundance and economic growth. NBER Working Paper, No5398, 54 p., Cambridge: National Bureau of Economic Research. December 1995. DOI: 10.3386/ w5398. Disponível em: <https://www.nber.org/papers/w5398.pdf>. Acesso em: 15 out. 2019.

SEAS AT RISK. European Commission and Azores question need for deep-sea mining, Feb. 2018. Disponível em Seas-at-risk.org: <https://seas-at-risk. org/27-deep-sea-mining/836-european-commissionand-azores-question-need-for-deep-sea-mining.html> . Acesso em: 10 março 2019.

SEAS AT RISK. European Parliament calls for international moratorium on deep-sea mining, January 2018. Disponível em: seas-at-risk.org/: <https:// seas-at-risk.org/27-deep-sea-mining/829-europeanparliament-calls-for-international-moratorium-on-deep-sea-mining.html>. Acesso em: 13 abril 2019.

SHUKMAN, David. Renewables' deep-sea mining conundrum. BBC News. Science \&Environment, 11 de abril de 2017. 1 vídeo (1 min.). Disponível em: $\quad<$ https://www.bbc.com/news/science-environment-39347620>. Acesso em: 15 out. 2018

SMITH, C. R.; DRAZEN, J.; MINCKS, S. L. Deep-sea Biodiversity and Biogeography: Perspectives from the Abyss. In: INTERNATIONAL SEABED AUTHORITY SEAMOUNT BIODIVERSITY SYMPO- 
SIUM, Honolulu, Hawaii USA, 2006.Pág. 2. Disponível em: <https://www.soest.hawaii.edu/oceanography/ mincks/publications/Smith_etal_Abyssal_biogeography_synthesis.pdf $>$. Acesso em: 20 out. 2018.

SOLOMON ISLANDS. Consolidated Legislation (1978). The Constitution of Solomon Islands. Statutory Instruments. 1978, No. 783. Pacific Islands. The Solomon Islands Independence Order 1978. 31st May 1978. Laid before Parliament - 8th June 1978. Coming into Operation - 7th July 1978.

SPC (PACIFIC COMMUNITY)- EU (EUROPEAN UNION). Deep Sea Minerals Project. Pacific ACP States. About The SPC-EU Deep Sea Minerals Project. Disponível em: <http://dsm.gsd.spc.int/>. Acesso em: 11 out. 2018.

STEINER, Richard (Professor). Independent Review of the Environmental Impact Statement for the proposed Nautilus Minerals Solwara 1 Seabed Mining Project.Madang, Papua New Guinea. 27 p. 10, Jan. 2009. Disponível em: <http://www.deepseaminingoutofourdepth.org/wp-content/uploads/SteinerIndependent-review-DSM1.pdf $>$. Acesso em: 20 out. 2018. < http://www.scoop.co.nz/stories/WO1810/ S00078/nautilus-are-you-nuts.htm>

SWADDLING Alison. Pacific-ACP States, Regional environmental management framework for deep sea minerals exploration and exploitation. Prepared under the SPC-EU EDF 10 Deep Sea Minerals Project by Alison SWADDLING, Environment Advisor. Suva, Fiji. June 2016. 100 p. Disponível em: <http://dsm.gsd. spc.int/images/public_files_2016/REMF2016.pdf>. Acesso em: 15 out. 2018. ISBN: 978-982-00-1007-9.

UNITED STATES DISTRICT COURT FOR THE DISTRICT OF COLUMBIA. Complaint for declaratory and other relief. May 2015. 21 pp. Disponível em: <https://www.biologicaldiversity.org/campaigns/ deep-sea_mining/pdfs/Deep-seabedMiningComplaint_05-12-2015.pdf>. Acesso em: 11 março 2019.

THE WORLD BANK (UNITED NATIONS DEPARTMENT OF ECONOMIC AND SOCIAL AFFAIRS). The Potential of the Blue Economy : Increasing Long-term Benefits of the Sustainable Use of Marine Resources for Small Island Developing States and Coastal Least Developed Countries. The World Bank, International Bank for Reconstruction and Development. Washington DC: World Bank, 2017. Disponível em: <https://openknowledge.worldbank. org/bitstream/handle/10986/26843/115545. pdf ? sequence $=1$ \&isAllowed $=\mathrm{y}>$. Acesso em: 10 abril 2019.

THE WORLD BANK. Pacific Possible. Precautionary Management of Deep Sea Mining Potential in Pacific Island Countries. Washington DC: World Bank, 2016. Disponível em: <http://pubdocs. worldbank.org/en/125321460949939983/Pacific-Possible-Deep-Sea-Mining.pdf>. Acesso em: 10 abril 2019.

TRIBUNAL INTERNATIONAL DU DROIT DE LA MER. Affaire n ${ }^{\circ} 17$ - Responsabilités et obligations des États qui patronnent des personnes et des entités dans le cadre d'activités menées dans la Zone (Avis consultatif - Chambre pour le règlement des différends relatifs aux fonds marins). Disponível em: https://www. itlos.org/fr/affaires/role-des-affaires/affaire-no-17/. Acesso em: 22 out. 2018.

ZHANG Denghua. China looking under the sea for opportunities in the Pacific. Papua New Guinea Mine Watch. 30 June 2018. Disponível em: < https://ramumine.wordpress.com/2018/07/02/china-looking-under-the-sea-for-opportunities-in-the-pacific/>. Acesso em: março 2019. 
Para publicar na Revista de Direito Internacional, acesse o endereço eletrônico www.rdi.uniceub.br ou www.brazilianjournal.org.

Observe as normas de publicação, para facilitar e agilizar o trabalho de edição. 